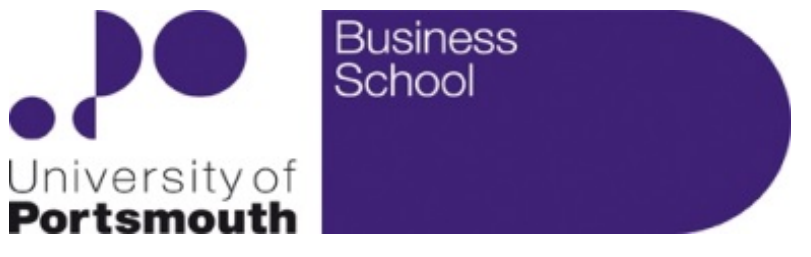

Working Papers in Economics \& Finance

2018-05

\title{
Spoofing and Pinging in Foreign Exchange Markets
}

\author{
Alexis Stenfors, University of Portsmouth \\ Masayuki Susai, Nagasaki University
}




\title{
Spoofing and Pinging in Foreign Exchange Markets
}

\author{
Alexis Stenfors $^{* *}$ and Masayuki Susai*
}

13 September 2018

\begin{abstract}
This paper investigates the susceptibility of FX spot markets to limit order submission strategies that are either intended to create a false impression of the state of the market ('spoof orders') or to extract hidden information in the market ('ping orders'). Using a complete limit order book dataset from EBS, we study currency pairs that have mature algorithmic markets (EUR/USD and USD/JPY), as well as other G10 and emerging market currencies where EBS is used as a secondary electronic trading platform (EUR/SEK, USD/RUB and USD/TRY). Our results, indicating that EUR/USD and USD/JPY are highly sensitive to information-rich orders, suggests that spoofing tactics might be more dependent on the chosen electronic trading venue, rather than the overall market liquidity of the currency pairs. Furthermore, we find widespread adoption of pinging tactics in the EUR/SEK and USD/RUB markets.
\end{abstract}

JEL Classification Numbers: D4, F3

Keywords: market microstructure, limit order book, foreign exchange, high-frequency trading, manipulation, spoofing, pinging, stealth trading

** Alexis Stenfors (corresponding author), University of Portsmouth, Richmond Building, Portland Street, Portsmouth PO1 3DE, UK. Telephone: +44 (0)23 9284 4183. E-mail: alexis.stenfors@port.ac.uk

${ }^{*}$ Masayuki Susai, Nagasaki University, 4-2-1, Katafuchi, Nagasaki, 850-8506 Japan. Telephone: +81 (0)95 820 6356. E-mail: msusai@nagasaki-u.ac.jp 


\section{Introduction}

Following the 'Forex scandal', the regulatory landscape in over-the-counter (OTC) markets is rapidly changing. However, human foreign exchange (FX) traders are not alone in having been caught up in the controversies hitherto resulting in fines exceeding \$10 billion - largely involving manipulation and misconduct in relation to orders submitted by banks' clients. Behaviour on electronic trading platforms has also come under scrutiny. In 2015, Barclays, BNP Paribas and Credit Suisse were fined for their use of a "Last Look" system on their single-bank trading platforms. ${ }^{1}$ Designed to reject potentially toxic order flow from high-frequency traders, their systems had been extended to distinguish which customer trades would be potentially (un)profitable for the bank. Furthermore, in 2017, the US Department of Financial Services fined Credit Suisse \$135 million for 'unlawful, unsafe and unsound' FX trading conduct. The consent order also mentioned 'front-running', whereby the bank had designed an inhouse trading algorithm to front-run limit orders and stop-loss orders submitted by clients (DFS, 2015; 2017ab).

In parallel, the attention to electronic limit order books is growing due to new legislation, most notably the 2010 Dodd-Frank Act, which explicitly makes 'spoofing' a criminal act in the commodities and futures markets. The convictions and regulatory settlements so far suggest that markets highly populated with algorithmic limit orders could be particularly susceptible to manipulative trading tactics such as spoofing (CFTC, 2018). Although not legally binding, the new Global FX Code stresses that 'market participants should not [...] create orders with the intention of disrupting market functioning or hindering the price discovery process, including undertaking actions designed to result in a false impression of market price, depth, or liquidity' (BIS, 2017).

The purpose of our paper is to explore the susceptibility of markets to limit order submission strategies that either intended to create a false impression of the state of the market ('spoof orders') or to extract hidden information in the market ('ping orders'). Although most jurisdictions do not explicitly outlaw spoofing in the OTC

\footnotetext{
${ }^{1}$ According to the BIS (2016), electronic, rather than voice-based, FX trading amounted to $57 \%$ in 2016. Electronic FX trading is, in turn, split between direct (e.g. single-bank platforms) and indirect
} 
FX markets, they are widely considered 'unethical'. 'Pinging', by contrast, has not received the same amount of scrutiny from regulators and lawmakers. However, the practice, involving ultra-frequent submissions and cancellations of limit orders, is by no means uncontroversial.

With a daily turnover of $\$ 5.1$ trillion, the global FX is, by far, the largest market in the world. However, mainly being a banking activity, it is also notoriously opaque and complete limit order book data has been, and remains, difficult to obtain. Thus, a vast and growing bulk of the market remains an unexplored area in the academic literature. Having acquired a full limit order book dataset provided by EBS, our paper aims to break this tradition. Importantly, our dataset contains a very high proportion of limit orders in comparison to market orders $(>99 \%)$. To enable a cross-market comparison, we study currency pairs having mature algorithmic markets on EBS (EUR/USD and $\mathrm{USD} / \mathrm{JPY}$ ), as well as other G10 and emerging market currencies where EBS is mainly used as a secondary electronic trading platform by traders (EUR/SEK, USD/RUB and USD/TRY). Our results, indicating that EUR/USD and USD/JPY are highly sensitive to information-rich orders, suggests that spoofing tactics might be more dependent on the chosen electronic trading venue, rather than the overall market liquidity of the currency pairs. Furthermore, we find widespread adoption of pinging tactics in the EUR/SEK and USD/RUB markets.

Our paper contributes to the empirical FX market microstructure literature hitherto overwhelmingly conducted on market orders or non-algorithmic settings (e.g. Daniélsson et al., 2012; King and Rime, 2010; Payne, 2003). We have also drawn inspiration from the survey-based work conducted by Cheung and Wong (2000) and Cheung and Chinn (2001), where the authors document unique behavioural aspects and social norms in the FX markets. Our study could also be seen in the context of the growing body of articles on deceptive limit order submission behaviour such as spoofing (e.g. Lee et al., 2012) and dark pools in exchange-traded markets (Bessembinder et al., 2009; Bloomfield et al., 2015). Ultimately, we hope that our work will add to the on-going debate about effective regulation of OTC markets.

The structure of the paper is as follows. Section 2 provides a broad overview of the related literature. Section 3 describes the dataset. Section 4 studies limit order 
submissions in terms of price aggressiveness, size and order-splitting strategies, and examines the immediate reaction to such orders. Section 5 investigates order cancellations through the lens of non-execution and free-option risk, and the lifetime of limit orders. By doing so, it considers the prevalence of pinging in electronic FX markets. Section 6 concludes.

\section{Related Literature}

The academic literature on spoofing, pinging and other manipulative or controversial practices in FX markets is still minimal. There are several contributing factors to this relative absence.

First, when the Forex scandal broke in 2013, the market was still widely perceived to be too large and competitive to withstand successful attempts of manipulation. 'I'm sceptical of the ability of traders to manipulate the major currencies in a meaningful way given the massive size of this market [...] Governments themselves often have a difficult time moving foreign-exchange markets through their interventions,' an academic is quoted having said in a Bloomberg article with the headline 'Traders Said to Rig Currency Rates to Profit Off Clients' in 2013 (Vaughan et al., 2013). This perception, of the market as being virtually immune to deceptive practices, echoes the narrative of the international money market and the subsequent 'LIBOR scandal' (Stenfors, 2018; Stenfors and Lindo, 2018).

Second, unlike securities markets, FX markets have, until recently, remained overwhelmingly unregulated. Consequently, whereas manipulative practices have been in breach of regulation (or even illegal) for equities and other securities, other rules have applied to FX markets. Instead, norms and conventions about what would constitute unfair or unethical trading practices have evolved informally as a result of discussions among market-making banks - often in dialogue with end-users and central banks (Cheung and Wong, 2000; Cheung and Chinn, 2001; Stenfors, 2018; Stenfors and Susai, 2018a). 
Third, being decentralised, highly opaque and overwhelmingly a banking activity, there is no exchange or institution gathering and providing real-time price, volume and limit order data on the global $\$ 5.067$ trillion-a-day OTC FX market (BIS, 2016). The closely related literature, therefore, mainly falls into one of two categories. The first includes market microstructure papers either focusing on limit orders in the stock market or, alternatively, empirical studies using transaction data, indicative prices, or incomplete order book samples in the FX market. The second category encompasses articles within law and business ethics, and reports published by policymakers since the Forex scandal.

The market microstructure literature addresses order submissions and the price determination process in detail. This often includes the strategic behaviour of traders and its impact on the state of the market. It uses the following logic. Market orders, which are intended to be executed immediately, contain information. Everything else being equal, a buy [sell] order submitted by a trader is more likely to be interpreted as causing the price to increase [decrease]. Consequently, order flow has an impact on the direction of the FX price movement (Lyons, 1997; Evans and Lyons, 2002). This relationship has been shown to hold, at least in the short run (see, for instance, Evans and Lyons, 2005; King and Rime, 2010; Payne, 2003). ${ }^{2}$

However, market participants also submit limit orders in the FX market, which are not intended to be executed immediately. A limit order submission involves a continuing assessment of the market in relation to the limit order submission. If the market moves away, the trader runs the risk of not trading at all (non-execution risk) (Fong and Liu, 2010; Liu, 2009). Alternatively, the trader might be picked off (free-option risk). In line with a surge in the proportion of limit orders following the rise of algorithmic trading in equity markets, limit orders have become seen as central to strategic trading (Foucault, 1999; Foucault et al., 2005; Rosu, 2009).

However, limit order submissions do not merely require post-submission monitoring. The submission itself involves a strategic trade-off between size and price

\footnotetext{
${ }^{2}$ Key empirical work on the price impact of FX order flow has been conducted using transaction data from major market-making banks, e.g. Citibank (Fan and Lyons, 2003), RBS (Osler and Vandrovych, 2009) and State Street Corporation (Froot and Ramadorai, 2002).
} 
aggressiveness to avoid front-running by other traders (Lo and Sapp, 2010). What is more, several studies (mostly conducted on equity markets) show that traders adopt stealth trading and order-splitting strategies in attempts to disguise the 'true' size of the limit order (Baclay and Warner, 1993; Engle et al., 2012; Chan and Lakonishok, 1995; Chou and Wang, 2009; Pérold 1988; Yeo, 2005). By being faster than humans, algorithmic traders have an advantage in slicing orders into smaller pieces to reduce the price impact and the transaction costs resulting from disappearing liquidity (Bertsimas and Lo, 1998). However, despite being designed to "hide" information, split orders are not regarded as deceptive limit order submission strategies.

A spoof order, by contrast, is deceptive from the start. The 2010 Dodd-Frank Act outlaws spoofing in the commodities and futures markets, and defines the practice as 'bidding or offering with the intent to cancel the bid or offer before execution'. The convictions and regulatory settlements so far suggest that markets highly populated with algorithmic limit orders could be particularly susceptible to spoofing, as the tactic benefits greatly from speed (CFTC, 2018). Although most jurisdictions do not explicitly outlaw spoofing in the OTC FX markets ${ }^{3}$, the practice is nonetheless considered unethical as it is intended to create a misperception of the state of the market.

From a microstructure perspective, spoofing can be viewed from the mirrored perspective of free-option risk and non-execution risk. A spoof order is intended to immediately trigger cancellations by traders on the opposite side of the limit order book (free-option risk by others). Similarly, a spoof order is intended to trigger submissions by traders on the same side of the limit order book (non-execution risk by others). Put differently, the aim with a spoof order is to cause other traders to react as if genuine price-moving information has entered the market.

Ultimately, what Allen and Gale (1992) refer to as 'trade-based manipulation' (and other forms of deceptive practices) has a strategic underpinning. According to Pirrong (2017), price manipulation can be defined as 'intentional conduct that causes market prices to diverge from their competitive level'. Numerous other definitions exist for

\footnotetext{
${ }^{3}$ However, according to Queree (2016), the tactic might be covered under certain fraud legislations already.
} 
manipulative conduct. However, the general view is that to prove that a trader has attempted to manipulate a price, two factors need to be established. First, the trader needs to have had the intention to move the price in a direction that is not consistent with normal market forces. Second, the trader needs to have taken actions to further that intent. ${ }^{4}$ Spoof orders influence other traders' perception of the supply and demand in the market (and consequently their view of the likely future price movement) and is, therefore, a form of market manipulation (Cumming et al., 2011; Cumming et al., 2015).

Pinging, by contrast, has not received the same amount of scrutiny by regulators and lawmakers. However, the practice is by no means uncontroversial. Pinging involves submitting a limit order very fast. If nothing happens, the order is immediately cancelled. If something happens, the trader extracts 'hidden' information from the limit order book, which can then be taken advantage of (McGowan, 2010). Ping orders are often referred to in relation to dark pools. In such a setting, pinging might be used by high-frequency traders to detect large (but hidden) orders with the intention to front-run them. ${ }^{5}$ However, with the term originating from naval sonar pings, the tactic is also applicable to partly or wholly visible limit order books.

The harshest critics label pinging as a form of automated and legalised front-running, emphasising the ability to obtain market-moving information, which can then be acted upon before other (human) traders. Front-running, itself, is a relatively broad term that involves trading in front of a large customer market or limit order to benefit from the expected price movement the order is likely to generate as a result of being executed in the market. Being a form of market manipulation, front-running is prohibited in most regulated securities markets. However, being both largely unregulated and challenging to enforce, the practice has hitherto not been explicitly illegal in the global FX market. ${ }^{6}$ However, in July 2016, two FX spot traders at HSBC were

\footnotetext{
${ }^{4}$ Importantly, a profit to the trader (or a loss to another) as a result of the manipulative scheme is not a prerequisite (CFTC, 2014).

${ }^{5}$ In comparison to equity markets, "dark pools" are still rare in the global FX market (BIS, 2016).

${ }^{6}$ The Swiss Financial Market Supervisory Authority FINMA (2014) referred to front running several times in its FX investigation and subsequent fine imposed to UBS of Sfr134 million. A year later, FINMA (2015) issued industry bans for between one and five years against six FX and precious metals traders for, among other things, engaging in front running.
} 
charged with conspiring to defraud a client through front-running on behalf of the bank (DOJ, 2016). ${ }^{7}$

Being overwhelmingly a decentralised OTC market, the FX market-making function has traditionally been performed by banks voluntarily - acting as principals and agents simultaneously. This increases the risk of manipulative conduct, as the private information obtained through orders gives large banks an advantage in predicting exchange rate movements (Peiers, 1997). Indeed, although a limit order, in contrast to a market order, is not executed immediately, it contains information about the likely future direction of the price. For instance, clients in the FX market frequently leave 'stop-loss orders' and 'take-profit orders' to market makers. By instructing the bank to buy or sell a specific amount of a currency at a specific price, the client can rest assured that the loss is limited or the profit is guaranteed should the market price reach a pre-defined level. ${ }^{8}$ A substantial in-house order book can, therefore, provide valuable (and confidential) information about the balance between supply and demand in the market, as well indications with regards to the sensitivity of the market to specific price levels. The Forex scandal involved many cases where conflicts of interest stemming from FX traders acting as both agents (receiving limit orders from clients) and principals (submitting market/limit orders on behalf of the bank). 'Triggering a stop-loss' is a manipulative tactic involving intentional buying [selling] activity by a bank to push the market price higher [lower] so that the client order gets triggered. Attempts to trigger clients' stop-loss orders featured in all FX-related Final Notices issued by the FCA (2014) in November 2014 when it fined Citi, HSBC, JP Morgan Chase, RBS and UBS $£ 1.1$ billion in total for their FX spot practices.

Other observers, however, argue that pinging is distinctively different from frontrunning practices. Instead, ping orders are claimed to be no different from than other legitimate limit orders. With such a view, pinging might even be beneficial for the market as a whole as it helps to disseminate information and speeds up the price discovery process (Scorpino, 2015). This logic is consistent with those maintaining

\footnotetext{
${ }^{7}$ In this first criminal FX front-running case, one defendant was sentenced to two years in prison in April 2018. An extradition order for the other defendant was blocked by the UK Court of Appeal in July 2018 (Hodges, 2018).

${ }^{8}$ Osler $(2003 ; 2005)$ shows that stop-loss orders often have a tendency to cluster around even numbers and can result in self-reinforcing price movements when large positions are unwound.
} 
that the liquidity provision by high-frequency traders enhances overall market quality (Bershova and Rakhlin 2013; Brogaard et al., 2014; Conrad et al., 2015; Hendershott et al., 2011).

Anecdotal evidence we have gathered from market participants points to there also being a third view. Many FX spot traders actively using EBS (and other platforms) argue that pinging, despite being legal, "does not add anything to the market", or even "misrepresents the true market". The concern that high-frequency trading, by crowding out the human market-making function, might result in unknown systemic consequences from a liquidity perspective is not new (BIS, 2011, BIS, 2017, Federal Reserve 2017, Harris, 2013, Stenfors and Susai, 2018a). However, should ping orders act to create an artificial view of the state of the market, they could be seen as directly harmful. Indeed, Scorpino (2015) argues that pinging should not be analysed within the framework of front-running. Instead, they should be viewed in the context of 'disruptive, manipulative, or deceptive trading practices', which are illegal in most securities markets (such as 'banging the close', 'spoofing' and 'wash trading').

\section{Data}

We use a high-frequency dataset from 21:00:00 (GMT) on 8 September 2010 to 20:59:59 (GMT) on 13 September 2010 (excluding the weekend) obtained from EBS. ${ }^{10}$ The share of algorithmic trading on EBS, together with Reuters Matching the most widely used platform used by market-making banks, increased from 2\% in 2004 to around $70 \%$ in 2013 (Moore et al., 2016). On EBS, traders can either submit a market order or a limit order. In addition to indicators of market orders, limit orders and transactions, we also use the price, volume, the buy or sell indicator and the

\footnotetext{
${ }^{9}$ A significant portion of the FX scandal related to practices labelled as banging the close in relation to fix orders. The most widely used FX benchmark is the WM/Reuters 4 p.m. fix, which is based on actual FX trading by market participants just before and just after the time of fixing (the 'fixing window'). A bank accepting a client buy [sell] order for a specific amount at the fix rate agrees to sell [buy] that amount at the fix rate, regardless where the market price is after that. Banging the close could involve a manipulative strategy whereby a trader attempts to influence the underlying price, typically very close to the time of fixing, to profit from the fix order. Thus, the logic is similar to manipulating a market, which underpins the benchmark for a derivatives position. As the value of a derivative depends on the outcome of the fixing, the payoff is directly affected by the manipulation of the underlying market (Jarrow, 1994; Pirrong, 2017). Attempts to manipulate the WM/Reuters 4 p.m. fix and other frequently used FX benchmarks feature in several of the settlements between banks and the UK and US authorities.

${ }^{10}$ We use the same dataset as in Stenfors and Susai (2018b).
} 
millisecond timestamp of each entry. A unique 20-digit Trader ID is attached to each indicator, allowing us to match order submissions with their order cancellations. However, the identities or institutions are not revealed. We study five currency pairs: three G10 currency pairs (EUR/USD, USD/JPY and EUR/SEK) and two emerging market currency pairs (USD/RUB and USD/TRY) (See Table 1).

Table 1: Overview of the markets

\begin{tabular}{llllll}
\hline Currency pair & BIS rank & Group & Total limit order amount & Total market order amount & Main ECN \\
EUR/USD & 1 & G10 & $€ 1,818,803,000,000$ & $€ 16,793,000,000$ & Yes \\
USD/JPY & 2 & G10 & $\$ 1,020,022,000,000$ & $\$ 6,518,000,000$ & Yes \\
EUR/SEK & 11 & G10 & $€ 52,839,000,000$ & - & No \\
USD/RUB & 12 & EM & $\$ 212,680,000,000$ & - & No \\
USD/TRY & 16 & EM & $\$ 38,704,000,000$ & - & No \\
\hline
\end{tabular}

Sources: EBS, BIS (2013) and authors' calculations.

The US dollar, the euro and the Japanese yen are the three most actively traded currencies, whereas the Swedish krona was ranked $11^{\text {th }}$ in terms of market turnover in 2013. The Russian rouble and the Turkish lira are two of the most actively traded emerging market currencies and were ranked $12^{\text {th }}$ and $16^{\text {th }}$ regarding global FX turnover in 2013 (BIS, 2013). EBS is the most widely used electronic trading platform among market-making banks for the major currency pairs and a selection of others. Reuters Matching, however, is the preferred platform for EUR/SEK and USD/TRY. USD/RUB is more liquid on the Moscow Exchange (MOEX) within the domestic market. $^{11}$

In line with the growth of algorithmic trading, more than $99 \%$ of all orders for all currency pairs in our dataset are limit orders. After having removed a small number of limit order submissions that do not contain a corresponding cancellation within the same day, the total number of limit orders in our study amounts to 2,330,480 with a total value of more than $\$ 3$ trillion. As can be seen, during the period of study, market orders were only submitted in currency pairs where EBS is considered the leading platform. Nonetheless, a considerable number of limit orders were submitted in all currency pairs.

\footnotetext{
${ }^{11}$ As detailed market shares are not publicly available, we have consulted with FX spot traders in each currency pair at market-making banks.
} 
There is almost an equal number of buy and sell orders (see Table 2). This symmetry is typical in the FX market as it is not subject to an intra-day buy-bias like the stock market.

Table 2: Overview limit order submissions and price range

\begin{tabular}{llllll}
\hline Currency pair & Buy orders (\%) & Sell orders (\%) & Low & High & Price range (\%) \\
EUR/USD & $50.65 \%$ & $49.35 \%$ & 1.2643 & 1.2893 & $1.96 \%$ \\
USD/JPY & $49.69 \%$ & $50.31 \%$ & 83.49 & 84.50 & $1.20 \%$ \\
EUR/SEK & $51.12 \%$ & $48.88 \%$ & 9.1600 & 9.2540 & $1.02 \%$ \\
USD/RUB & $50.26 \%$ & $49.74 \%$ & 30.64 & 30.97 & $1.07 \%$ \\
USD/TRY & $51.37 \%$ & $48.63 \%$ & 1.4866 & 1.5204 & $2.25 \%$ \\
\hline Sources: EBS and authors' calculations. Notes: 'Low' and 'high' denotes the lowest and highest mid-market limit order price \\
during 8-13 September 2010.
\end{tabular}
during 8-13 September 2010.

With a price range of approximately $1-2 \%$ for all currency pairs, the period ought to be suitable for a cross-country study under relatively comparable market conditions. Furthermore, by selecting a group of currencies covering both G10 and emerging markets, with different conventions relating to electronic trading, we hope to be able to capture some unique characteristics. Perhaps most importantly, the full limit order book dataset enables a line of inquiry that differs significantly from previous studies based upon transaction data or the era when the FX market was still predominantly conducted by human traders.

\section{$4 \quad$ The Susceptibility to Spoofing}

\subsection{The Sensitivity to Aggressive Limit Orders}

Market orders are intended to be executed immediately and are therefore submitted at a price, which is aggressive enough to ensure an immediate execution. Limit buy [sell] orders (including spoof orders), by contrast, are strategically placed at some distance from the prevailing best ask [bid] price in the market. The aim with a spoof is to cause other traders to react as if genuine price-moving information has entered the market. Price aggressiveness is therefore key, as an aggressive order is likely to be interpreted as more information-rich by others. Thus, a 'successful spoof' would display similar properties as a limit order submission, which is cancelled due to nonexecution risk. The critical difference lies in the intent. Whereas a legitimate limit order would be cancelled as a result of an increase in the distance between the market 
and the limit order submission price (and then perhaps resubmitted at a more aggressive price level), the purpose of a spoof order would be to trigger such a change (without an intent to execute a trade).

Thus, let us first investigate if and how traders on the electronic platform for the five currency pairs immediately react to aggressive limit order submissions by others.

To do so, we run the following model:

$R_{i j}=\alpha_{i}+\beta_{1} M_{-} A_{i}+\beta_{2} M_{-} L_{i}+\beta_{3} V_{0 l} l_{i}+\beta_{4} B_{-} A_{i}+\delta_{1} P A_{-} 9-10_{i}+\delta_{2} P A_{-} 8_{i}+\delta_{3} P A_{-} 7_{i}+$ $\delta_{4} P A_{-} 6_{i}+\delta_{5} P A_{-} 5_{i}+\varepsilon_{i}$

In Equation 1, we define four dependent variables $\left(R_{j}\right)$ as follows:

- $R_{1}$ : Opposite-side limit order cancellation

- $R_{2}$ : Same-side limit order submission

- $R_{3}$ : Same-side limit order cancellation

- $R_{4}$ : Opposite-side limit order submission

If limit order submission $i$ is immediately followed a reaction $j$ (where $j=1,2,3$ or 4 ), then $R_{j}$ takes the value of 1 , otherwise 0 .

As limit orders are not intended to be executed immediately, the choice of price aggressiveness is critical. The further away from the current market price, the less likely a limit order will result in a trade. We classify the limit order submissions according to their price aggressiveness as follows:

- $P A \_10$ : if the new limit order submission price $p_{i}$ improves the current best bid-ask spread (i.e. a marketable limit order).

- $P A \_9$ : if the new limit order submission price $p_{i}$ matches the current best bidask spread (i.e. an at-the-quote limit order)

- $P A \_8$ : if the new limit order submission price $p_{i}$ is outside, but within [EUR/USD, USD/JPY: 1 pip; USD/TRY: 2 pips; EUR/SEK, USD/RUB: 25 pips] of, the best bid-ask spread.

- PA_7 : if the new limit order submission price $p_{i}$ is outside of [EUR/USD, USD/JPY: 1 pip; USD/TRY: 2 pips; EUR/SEK, USD/RUB: 25 pips] from, but within [EUR/USD, USD/JPY: 2 pips; USD/TRY: 4 pips; EUR/SEK, USD/RUB: 50 pips] of, the best bid-ask spread. 
- $[\ldots]$

- $P A_{-} 1$ : if the new limit order submission price $p_{i}$ is at least [EUR/USD, USD/JPY: 7 pips; USD/TRY: 14 pips; EUR/SEK, USD/RUB: 175 pips] from the best bid-ask spread. ${ }^{12}$

In our model, we use dummy variables to capture the higher levels of price aggressiveness (5 to 10 ). Thus, the baseline limit order has a price aggressiveness level of 4 or below. To obtain a sufficient number of observations for all currency pairs at all levels of aggressiveness, we also combine marketable and at-the-quote limit orders (PA_9-10).

We also use a string of control variables as follows:

- $\quad M \_A=$ Let us define the $i$ th limit order submission as $L O S_{i}$. As a proxy for market activity, where $M_{-} A_{i}=\sum_{j=t(i)-60 s}^{j=t(i)} L O S_{j}$, we use the number of limit orders submitted to the EBS platform in the respective currency pair during the previous 60 seconds.

- $M_{-} L$ : The market liquidity at the current best bid-ask spread, where $M_{-} L_{i}=\sum_{j=t(0)}^{j=t(i-1)} A_{j}^{b b}+\sum_{j=t(0)}^{j=t(i-1)} A_{j}^{b a}$, where $A^{b b}\left[A^{b a}\right]$ is the amount of outstanding limit buy [sell] orders at best bid [ask] and $t(0)=21: 00: 00$ GMT.

- $\quad B \_A$ : The bid-ask spread, i.e. the difference between the best ask and bid prices, $\left(p_{t(i-1)}^{b a}-p_{t(i-1)}^{b b}\right)$ measured vis-à-vis the mid price, $p_{t(i-1)}^{b m}$, on the EBS platform immediately prior to the limit order submission. Thus, $B_{-} A_{i}=\left(p_{t(i-1)}^{b a}-p_{t(i-1)}^{b b}\right) / p_{t(i-1)}^{b m}$.

- $\quad V o l$ : Volatility, which is measured using the mid-market price of the best limit buy and sell orders $\left(p^{b m}\right)$ at each second during a 60 -second interval prior to the new limit order submission, i.e. $V_{i}=$ $\left.\left.\sqrt{252 \times 24 \times 60 \times \sigma\left(\sum_{j=t(i)-61 s}^{j=t(i)-1 s}\left(\left(p_{j-1 s}^{b m}\right.\right.\right.} / p_{j-2 s}^{b m}\right)-1\right)$, where $\sigma$ is the variance.

As the dependent variables are binary, we use a probit model and estimate the four equations from $R_{l}$ to $R_{4}$ independently. For robustness checks, we divide the whole sample into three subsamples (as our dataset covers three trading days). We also run

\footnotetext{
${ }^{12}$ Note: 1 pip $=4$ th decimal for EUR/USD, EUR/SEK, USD/RUB and USD/TRY and 2nd decimal for USD/JPY. To account for different market conventions and currency values, as well as market size and liquidity, we employ a corresponding straight-line scale between $P A_{-} 1$ and $P A_{-} 8$.
} 
tests using and more (and fewer) levels of aggressiveness. However, the results are very similar. 


\subsection{Results}

The results are shown in Table 3.

Table 3: Results (Equation 1)

\begin{tabular}{|c|c|c|c|c|c|c|c|c|}
\hline \multirow{4}{*}{$\begin{array}{l}\text { EUR/USD } \\
\text { Mean dependent variable } \\
\text { Constant } \\
\text { M A }\end{array}$} & \multirow{2}{*}{\multicolumn{2}{|c|}{$\begin{array}{l}\text { Opposite-side cancellation } \\
0.211\end{array}$}} & \multirow{2}{*}{\multicolumn{2}{|c|}{ Same-side submission }} & \multirow{2}{*}{\multicolumn{2}{|c|}{ Same-side cancellation }} & \multirow{2}{*}{\multicolumn{2}{|c|}{ Opposite-side submission }} \\
\hline & & & & & & & 0.184 & \\
\hline & $-1.049^{* *}$ & $(0.004)$ & $-0.754 * *$ & $(0.004)$ & $-0.154^{* *}$ & $(0.003)$ & $-0.888^{* *}$ & $(0.004)$ \\
\hline & $0.025 * *$ & $(0.003)$ & $0.016^{* *}$ & $(0.003)$ & $-0.079 * *$ & $(0.003)$ & $0.057 * *$ & $(0.003)$ \\
\hline M_L & $0.867^{* *}$ & $(0.099)$ & $0.776^{* *}$ & $(0.093)$ & $-2.994^{* *}$ & $(0.095)$ & $1.847 * *$ & $(0.100)$ \\
\hline B_A & $0.724 * *$ & $(0.238)$ & $-5.939 * *$ & $(0.222)$ & $13.544^{* *}$ & $(0.224)$ & $-10.404 * *$ & $(0.243)$ \\
\hline Vol & -0.006 & $(0.007)$ & 0.008 & $(0.005)$ & -0.008 & $(0.005)$ & 0.005 & $(0.006)$ \\
\hline PA_9-10 & $0.356^{* *}$ & $(0.003)$ & $0.289^{* *}$ & $(0.003)$ & $-0.522 * *$ & $(0.003)$ & $-0.017^{* *}$ & $(0.004)$ \\
\hline $\mathrm{PA}_{-}^{-} 8$ & $0.237 * *$ & $(0.004)$ & $0.347^{* *}$ & $(0.003)$ & $-0.468^{* *}$ & $(0.003)$ & $-0.022^{* *}$ & $(0.004)$ \\
\hline PA_7 & $0.171 * *$ & $(0.005)$ & $0.266^{* *}$ & $(0.004)$ & $-0.395^{* *}$ & $(0.004)$ & $0.063 * *$ & $(0.005)$ \\
\hline PA_6 & $0.107^{* *}$ & $(0.006)$ & $0.135^{* *}$ & $(0.005)$ & $-0.210^{* *}$ & $(0.005)$ & $0.045^{* *}$ & $(0.005)$ \\
\hline PA_5 & $0.018^{* *}$ & $(0.006)$ & $0.161^{* *}$ & $(0.006)$ & $-0.240^{* *}$ & $(0.005)$ & $0.133^{* *}$ & $(0.006)$ \\
\hline McFadden R-squared & 0.009 & & 0.008 & & 0.022 & & 0.003 & \\
\hline Observations & \multicolumn{2}{|l|}{$1,419,659$} & & & & & & \\
\hline USD/JPY & \multicolumn{2}{|c|}{ Opposite-side cancellation } & \multicolumn{2}{|c|}{ Same-side submission } & \multirow{2}{*}{\multicolumn{2}{|c|}{$\begin{array}{l}\text { Same-side cancellation } \\
0.320\end{array}$}} & \multicolumn{2}{|c|}{ Opposite-side submission } \\
\hline Mean dependent variable & 0.211 & & 0.294 & & & & 0.175 & \\
\hline Constant & $-1.131 * *$ & $(0.006)$ & $-0.662^{* *}$ & $(0.005)$ & $-0.221 * *$ & $(0.005)$ & $-0.828^{* *}$ & $(0.005)$ \\
\hline M_A & $0.066^{* *}$ & $(0.006)$ & $0.012^{*}$ & $(0.006)$ & $-0.110^{* *}$ & $(0.006)$ & $0.061 * *$ & $(0.006)$ \\
\hline M_L & -0.046 & $(0.135)$ & $1.652^{* *}$ & $(0.125)$ & $-2.708^{* *}$ & $(0.127)$ & $1.447 * *$ & $(0.138)$ \\
\hline $\mathrm{B} A$ & 0.115 & $(0.235)$ & $-5.471^{* *}$ & $(0.221)$ & $10.745^{* *}$ & $(0.218)$ & $-7.470^{* *}$ & $(0.244)$ \\
\hline Vol & 0.040 & $(0.022)$ & 0.016 & $(0.026)$ & $-0.095^{* *}$ & $(0.024)$ & 0.043 & $(0.029)$ \\
\hline PA_9-10 & $0.468 * *$ & $(0.005)$ & $0.231 * *$ & $(0.004)$ & $-0.501^{* *}$ & $(0.004)$ & $-0.091^{* *}$ & $(0.005)$ \\
\hline PA 8 & $0.331 * *$ & $(0.007)$ & $0.230 * *$ & $(0.006)$ & $-0.403^{* *}$ & $(0.006)$ & $-0.051^{* *}$ & $(0.007)$ \\
\hline PA_7 & $0.294 * *$ & $(0.005)$ & $0.177^{* *}$ & $(0.005)$ & $-0.322 * *$ & $(0.005)$ & $-0.045^{* *}$ & $(0.005)$ \\
\hline PA_6 & $0.203^{* *}$ & $(0.010)$ & $0.051^{* *}$ & $(0.009)$ & $-0.144 * *$ & $(0.008)$ & $-0.039 * *$ & $(0.010)$ \\
\hline PA_5 & $0.090 * *$ & $(0.008)$ & $0.046^{* *}$ & $(0.007)$ & $-0.072^{* *}$ & $(0.006)$ & $-0.030 * *$ & $(0.007)$ \\
\hline McFadden R-squared & 0.014 & & 0.004 & & 0.019 & & 0.002 & \\
\hline Observations & 787,235 & & & & & & & \\
\hline EUR/SEK & Opposite-si & cancellation & Same-side su & mission & Same-side & cellation & Opposite-s & submission \\
\hline Mean dependent variable & 0.210 & & 0.032 & & 0.568 & & 0.190 & \\
\hline Constant & $0.342 * *$ & $(0.051)$ & $-0.925^{* *}$ & $(0.100)$ & $-1.592^{* *}$ & $(0.050)$ & $-0.271^{* *}$ & $(0.058)$ \\
\hline M_A & $-7.126^{* *}$ & $(0.299)$ & 0.162 & $(0.442)$ & $9.232 * *$ & $(0.255)$ & $-7.166^{* *}$ & $(0.314)$ \\
\hline M_L & $-343.798 * *$ & $(21.133)$ & $-635.998^{* *}$ & $(28.859)$ & $555.191 * *$ & $(21.108)$ & $-98.351 * *$ & $(25.058)$ \\
\hline B_A & $-2.415^{* *}$ & $(0.152)$ & $-4.055^{* *}$ & $(0.317)$ & $3.162 * *$ & $(0.137)$ & $-0.580^{* *}$ & $(0.168)$ \\
\hline Vol & $1.784 * *$ & $(0.120)$ & -0.094 & $(0.198)$ & $-2.333^{* *}$ & $(0.123)$ & $1.242^{* *}$ & $(0.139)$ \\
\hline PA_9-10 & $0.119^{* *}$ & $(0.027)$ & $0.958 * *$ & $(0.084)$ & $0.217 * *$ & $(0.025)$ & $-0.725^{* *}$ & $(0.029)$ \\
\hline PA_8 & $-0.843 * *$ & $(0.036)$ & $0.950 * *$ & $(0.087)$ & $0.810^{* *}$ & $(0.029)$ & $-0.720^{* *}$ & $(0.033)$ \\
\hline PA_7 & -0.036 & $(0.027)$ & $0.395^{* *}$ & $(0.089)$ & $-0.076^{* *}$ & $(0.025)$ & $0.084 * *$ & $(0.027)$ \\
\hline PA_6 & 0.053 & $(0.031)$ & $0.517^{* *}$ & $(0.093)$ & $-0.299 * *$ & $(0.028)$ & $0.245^{* *}$ & $(0.030)$ \\
\hline $\mathrm{PA}^{-} 5$ & $0.127^{* *}$ & $(0.031)$ & $0.475 * *$ & $(0.094)$ & $-0.549 * *$ & $(0.030)$ & $0.412 * *$ & $(0.030)$ \\
\hline McFadden R-squared & 0.073 & & 0.094 & & 0.122 & & 0.113 & \\
\hline Observations & 47,473 & & & & & & & \\
\hline USD/RUB & Opposite-si & cancellation & Same-side su & mission & Same-side & cellation & Opposite-s & submission \\
\hline Mean dependent variable & 0.263 & & 0.165 & & 0.352 & & 0.220 & \\
\hline Constant & $-0.590 * *$ & $(0.028)$ & $-0.299 * *$ & $(0.031)$ & $-0.637^{* *}$ & $(0.027)$ & $-0.945^{* *}$ & $(0.030)$ \\
\hline M_A & $-1.275^{* *}$ & $(0.490)$ & 0.198 & $(0.551)$ & $3.919^{* *}$ & $(0.467)$ & $-2.362^{* *}$ & $(0.507)$ \\
\hline $\mathrm{ML}$ & $9.024 * *$ & $(0.959)$ & $-51.389 * *$ & (1.358) & $14.250 * *$ & $(0.928)$ & $3.725^{* *}$ & (1.003) \\
\hline $\mathrm{B} A$ & $-1.798^{* *}$ & $(0.218)$ & $2.657^{* *}$ & $(0.163)$ & $-1.006^{* *}$ & $(0.186)$ & $-0.758^{* *}$ & $(0.160)$ \\
\hline Vol & 0.310 & $(0.213)$ & $0.672 * *$ & $(0.212)$ & $-1.377^{* *}$ & $(0.232)$ & 0.258 & $(0.217)$ \\
\hline PA_9-10 & $-0.141^{* *}$ & $(0.023)$ & 0.002 & $(0.025)$ & 0.026 & $(0.022)$ & $0.162 * *$ & $(0.025)$ \\
\hline PA_8 & $-0.078^{*}$ & $(0.037)$ & $-0.343^{* *}$ & $(0.047)$ & $0.087^{*}$ & $(0.035)$ & $0.267 * *$ & $(0.039)$ \\
\hline PA_7 & $-0.080^{* *}$ & $(0.026)$ & $-0.355^{* *}$ & $(0.030)$ & 0.033 & $(0.025)$ & $0.350 * *$ & $(0.028)$ \\
\hline PA_6 & 0.021 & $(0.038)$ & $-0.304 * *$ & $(0.047)$ & -0.074 & $(0.038)$ & $0.344 * *$ & $(0.040)$ \\
\hline PA_5 & $0.105^{* *}$ & $(0.029)$ & $-0.333^{* *}$ & $(0.034)$ & $-0.096^{* *}$ & $(0.029)$ & $0.291 * *$ & $(0.032)$ \\
\hline McF̄adden R-squared & 0.009 & & 0.098 & & 0.011 & & 0.009 & \\
\hline Observations & 31,778 & & & & & & & \\
\hline USD/TRY & Opposite-si & cancellation & Same-side su & mission & Same-side & cellation & Opposite-s & submission \\
\hline Mean dependent variable & 0.240 & & 0.101 & & 0.530 & & 0.129 & \\
\hline Constant & $-0.738 * *$ & $(0.040)$ & $-1.203^{* *}$ & $(0.053)$ & $0.234^{* *}$ & $(0.038)$ & $-1.415^{* *}$ & $(0.047)$ \\
\hline M_A & $-2.477 * *$ & $(0.182)$ & $1.224^{* *}$ & $(0.195)$ & $1.310^{* *}$ & $(0.148)$ & -0.213 & $(0.197)$ \\
\hline M_L & $124.309 * *$ & (14.191) & $-90.175^{* *}$ & $(18.851)$ & $-94.659^{* *}$ & (13.793) & $56.327 * *$ & (16.704) \\
\hline B_A & $-3.749^{* *}$ & $(0.234)$ & $-1.307^{* *}$ & $(0.315)$ & $3.293^{* *}$ & $(0.205)$ & 0.022 & $(0.263)$ \\
\hline Vol & -0.611 & $(0.435)$ & $-4.203^{* *}$ & $(0.630)$ & $3.823 * *$ & $(0.396)$ & $-4.527^{* *}$ & $(0.589)$ \\
\hline PA_9-10 & $0.133^{* * *}$ & $(0.018)$ & $-0.125^{* *}$ & $(0.026)$ & $-0.092^{* *}$ & $(0.017)$ & 0.044 & $(0.024)$ \\
\hline PA_ 8 & $0.109 * *$ & $(0.024)$ & $0.692 * *$ & $(0.029)$ & $-0.947 * *$ & $(0.024)$ & $0.715^{* *}$ & $(0.027)$ \\
\hline PA_7 & $0.073^{*}$ & $(0.034)$ & $0.617^{* *}$ & $(0.038)$ & $-0.897 * *$ & $(0.033)$ & $0.777^{* *}$ & $(0.035)$ \\
\hline $\mathrm{PA}_{-}^{-} 6$ & $-0.181 * *$ & $(0.043)$ & $0.650 * *$ & $(0.044)$ & $-0.567 * *$ & $(0.037)$ & $0.612 * *$ & $(0.042)$ \\
\hline PA 5 & $0.051^{*}$ & $(0.025)$ & $0.424 * *$ & $(0.030)$ & $-0.362^{* *}$ & $(0.023)$ & $0.278^{* *}$ & $(0.030)$ \\
\hline McFadden R-squared & 0.022 & & 0.067 & & 0.077 & & 0.054 & \\
\hline Observations & 38,502 & & & & & & & \\
\hline
\end{tabular}

Sources: EBS and authors' calculations. Notes: Binary probit model. */** denotes statistical significance at 95\%/99\% level. Standard errors in parentheses. M_A = Market activity $(* 1000)$, M_L = Market liquidity (bio), $\mathrm{Vol}=60$-second volatility $(\%), \mathrm{B} \_\mathrm{A}=$ Bid-ask spread $(\%), \mathrm{PA}=\overline{\text { Price }}$ aggressiveness. See Section 4.1 for detailed definitions of the variables.

Our filtered dataset contains more than 2.3 million limit order submissions and an equal number of cancellations. Furthermore, the proportion of buy/sell orders is also 
highly symmetrical and volatility relatively low (see Table 2). At the outset, therefore, it might be plausible to expect that the four reaction types by other traders (oppositeside cancellation, same-side submission, same-side cancellation or opposite-side submission) should be fairly equally distributed. However, as can be seen from the dependent variables, the immediate reaction to new limit order submissions does not follow a random process. Instead, a high level of clustering can be observed. There are significant differences among the currency pairs, irrespective of the level of price aggressiveness. In the EUR/USD and USD/JPY markets, approximately 50\% of new limit order submissions are followed by either an opposite-side cancellation or a same-side submission. In the EUR/SEK, USD/RUB and USD/TRY markets, however, the ratio is lower: $24 \%, 42 \%$ and $34 \%$, respectively.

As outlined previously, an aggressive limit buy [sell] order might trigger other traders to react by cancelling their sell [buy] orders (free-option risk) or submitting new, perhaps more aggressive, buy [sell] orders (non-execution risk). A more aggressive order should, in particular, be more likely to trigger an opposite-side cancellation than an opposite-side submission. A market sensitive to spoofing tactics ought to display similar characteristics. For spoofing tactics to be successful, market participants ought to react with some predictability: namely to immediately exit, rather than immediately enter, the opposite side of the limit order book.

As we can see from Table 3, the results support this logic for the EUR/USD and USD/JPY FX spot markets. A limit order submission with a higher level of price aggressiveness than the baseline order (i.e. $P A \_5$ or above) increases the likelihood of an opposite-side cancellation. What is more: the higher the level of price aggressiveness, the higher the probability of such an immediate reaction from other traders.

The coefficients for opposite-side submissions are more or less the reverse. This confirms that the higher the level of price aggressiveness, the less likely it is that the 
limit order submission is followed by a submission countered from the other side of the limit order book. Both results are strongly significant. ${ }^{13}$

The results for the EUR/SEK, USD/RUB and USD/TRY markets are, however, different and also inconsistent. Importantly, for some levels of price aggressiveness above 5, the coefficients are negative and strongly significant when testing for opposite-side cancellations. Thus, from the perspective of price aggressiveness, the two major currency pairs exhibit features consistent with markets susceptible to spoofing tactics. However, the same cannot be said about the three currency pairs where EBS typically acts as a secondary electronic trading platform (and to which we will return later).

\subsection{The Sensitivity to Order Size}

From studying price aggressiveness only, it appears as if the EUR/USD and USD/JPY markets are more susceptible to spoofing than the other three markets. However, a limit (and, indeed, a spoof) order submission involves a trade-off between price aggressiveness and size (Lo and Sapp, 2010; Lee et al., 2012). Theoretically, as well as anecdotally, a relatively large order would be interpreted as information-rich. A trader would, consequently, be inclined to carefully select the appropriate level of size and price aggressiveness to minimise the risk of front-running by other traders. Conversely, a spoof order would be constructed to increase the likelihood of an immediate cancellation from the opposite side of the order book. Thus, let us inspect this strategic trade-off in the limit order submission process.

The minimum order size on EBS is relatively large (\$/€ 1 million) as it is not a trading platform intended for retail investors. We separate the limit orders into three size categories:

\footnotetext{
${ }^{13}$ Theoretically, other traders should also react to aggressive limit buy [sell] order submissions by submitting new, perhaps more aggressive, buy [sell] orders (non-execution risk). Before they do so, however, they might need to cancel existing orders. In such a case, a cancellation, rather than submission, from the same side of the limit order book is the more logical reaction. The immediate reaction from the same side of the order book is therefore less clear. Nonetheless, the results suggest that same-side submissions, rather than cancellations, are more probable in the EUR/USD and USD/JPY markets.
} 
- Small : if the limit order size is equal to $\$ / € 1$ million

- Medium : if the limit orders size is greater than $\$ / € 1$ million but smaller than $\$ / € 5$ million

- Large : if the limit order size is equal to, or greater than $\$ / € 5$ million

However, knowing that a relatively large limit order submission is likely to trigger a stronger market impact, order-splitting strategies are frequently adopted. By slicing a larger order into smaller pieces, the "true" order size can thereby be disguised. As each limit order in our dataset contains an individual ID, we are unable to ascertain, with absolute certainty, which orders form part of such a strategy. Nonetheless, by adopting a string of very strict criteria, we can extract those, which are highly likely to be split orders. We define a 'split' (or, alternatively, 'independent') order as follows (see Stenfors and Susai, 2018b):

- Split : i) if the price of limit order submission $i, p_{t(i)}$, is the same as the price of limit order submission $j, p_{t(j)}$, where $j \neq i$ and $j>i$, ii) if the direction (i.e. buy or sell) of limit order submission $i$ is the same as the direction of limit order submission $j$, iii) if limit order $i$ and $j$ are submitted within less than 0.1 seconds of each other, and iv) if no other orders are submitted or cancelled in between the submissions of limit order $i$ and $j$.

- Independent $:$ if otherwise.

Figure 1 shows the distribution of limit orders according to size and order-splitting strategies for the five currency pairs.

Figure 1: Size and order-splitting

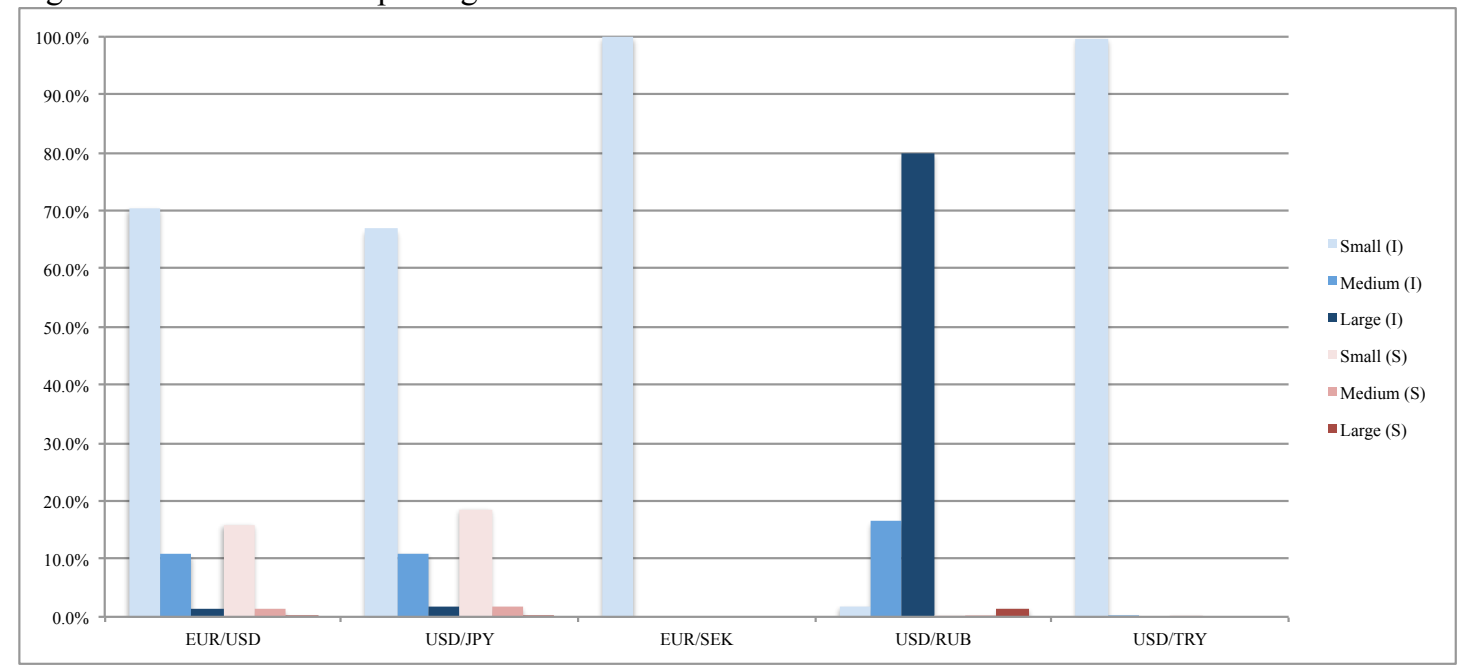

Sources: EBS and authors' calculations. Note: $\mathrm{S}=$ Split, I = Independent. 
As can be seen, the vast majority (over $85 \%$ ) of limit orders submitted in the EUR/USD and USD/JPY markets are for precisely $€ / \$ 1$ million. Even though these markets contain some substantial orders (the largest being $€ 250$ million in the EUR/USD market), less than $2 \%$ are classified as large. This is consistent with empirical findings of a "race-to-the-bottom" concerning order size in other markets increasingly populated with high-frequency traders (Biais et al., 1995; Hollifield et al., 2004). On EBS, however, the minimum order size rule puts a relatively stringent cap on this process - which serves to explain the clustering around $\$ / € 1$ million precisely.

Order splitting strategies to disguise true size appear to be very common in the EUR/USD and USD/JPY markets. Despite adopting stringent criteria, more than 400,000 split orders were submitted during just three trading days. There could be a logical reason for this. Being the largest and most liquid currencies, they have also witnessed the most significant influx of high-frequency and algorithmic traders which can submit such orders. Indeed, the prevalence of split orders lends support to the susceptibility of the markets for the two major currency pairs to spoofing. After all, the intent behind an order-splitting strategy is to avoid a reaction that a spoofing strategy is intended to cause. The sheer frequency of such orders suggests that market participants assume that other traders to react 'predictably' when price-moving information enters the market.

Classifying the order sizes and order-splitting strategies according to price aggressiveness illustrates this further. Figure 2 shows the average price aggressiveness for each order type the scale from 1 to 10 (see Section 4.1): 
Figure 2: Price aggressiveness, size and order-splitting

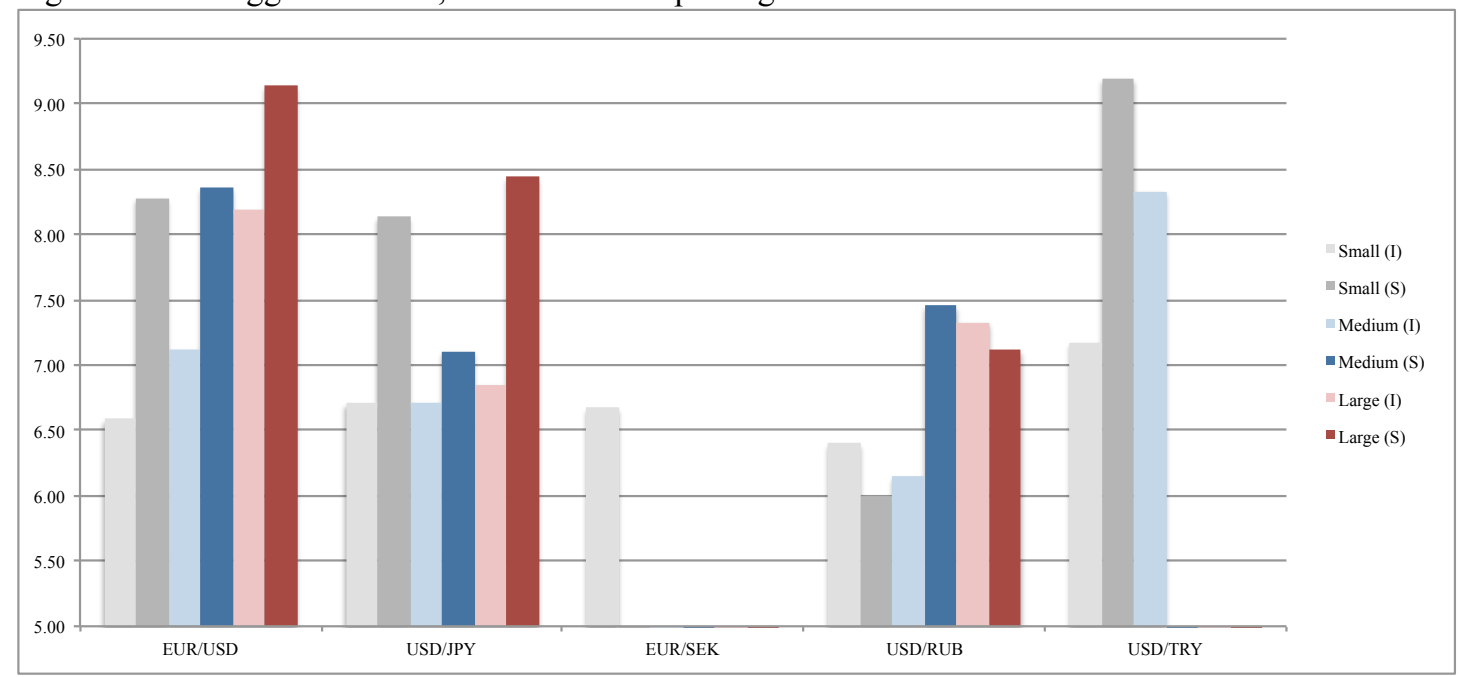

Sources: EBS and authors' calculations. Note: $\mathrm{S}=$ Split, I = Independent.

Interestingly, the results are inconsistent with Lo and Sapp (2010), who document a trade-off between size and price aggressiveness in the USD/DEM FX spot market using (human trader) data from 1997. In fact, medium-sized orders are considerably more aggressive than small orders (which are the norm) in the EUR/USD and USD/JPY markets. Large orders, too, are somewhat more aggressive than small orders.

There is, however, a clear trade-off between order-splitting and price aggressiveness in the EUR/USD and USD/JPY markets. Notably, small split orders (which count over 370,000 in our dataset) are, on average, submitted at $P A \_8$ or above significantly higher than small or medium-sized independent orders. Thus, the results confirm that order-splitting strategies enable algorithmic traders to submit more aggressive orders than otherwise (Keim and Madhavan, 1995, 1996). As ordersplitting could be seen as a means to minimise front-running by others, this would suggest that market participants, at least to some degree, perceive the two markets as susceptible to spoofing.

The results for the Swedish krona, the Russian rouble and the Turkish lira, where EBS typically acts as a secondary electronic trading platform, are, however, entirely different. In the EUR/SEK and USD/TRY markets, 100\% and $99.97 \%$ are limit orders of $€ / \$ 1$ million precisely. The USD/RUB market is, from the perspective of limit order size, an outlier. An overwhelming majority of limit orders submitted are at least 
\$5 million. Furthermore, given that USD/RUB has by far the highest proportion of relatively large limit orders in our study, we would expect this to be matched by a frequent adoption of order-splitting strategies. Indeed, using USD/RUB data from Moscow's MICEX exchange in 2002, Menkhoff and Schmelling (2010) find that informed traders engage in stealth trading through medium-sized orders. Surprisingly, we detect hardly any such activity. Employing somewhat less stringent criteria for split orders ( 0.2 rather than 0.1 seconds) changes the result only marginally. The share of split orders in the USD/RUB market increases from just $1.77 \%$ to $2.05 \%$. Notably, however, the datasets are very different. Menkhoff and Schmelling (2010) use exchange-traded transactions with an average order size of $\$ 50,000$ from a predominantly human-driven trading era. The average USD/RUB order size in our study is approximately 100 times larger and covers a period when algorithmic and high-frequency trading has established itself in the FX market. In the EUR/SEK and USD/TRY markets, order-splitting strategies are more or less completely absent. In sum, whereas the three currency pairs where EBS typically acts as a secondary electronic trading platform display limited susceptibility to spoofing, EUR/USD and USD/JPY warrant a closer inspection.

\subsection{An Extended Model}

In Section 4.2, we established that the higher the level of price aggressiveness of an EUR/USD or USD/JPY limit order submission, the higher the probability of triggering an immediate cancellation from the opposite side of the order book. In Section 4.3, however, we also noted that medium-sized and large orders, which due to their information content should trigger a similar reaction, tend to be relatively aggressive at the outset - as do split orders submitted by algorithmic traders to disguise such information. To test the impact of such trade-offs on immediate ordercancellations by others, we run the following probit model for $R_{l}$ : 
$R_{i 1}=\alpha_{i}+\beta_{1} M_{-} A_{i}+\beta_{2} M_{-} L_{i}+\beta_{3} V_{\text {Vol }}+\beta_{4} B_{-} A_{i}+\delta_{1} I_{i} \cdot \operatorname{Small}_{i} \cdot P A_{-} 9-10_{i}+\delta_{2} I_{i} \cdot \operatorname{Small}_{i} \cdot$ $P A_{-} 7-8_{i}+\delta_{3} I_{i} \cdot \operatorname{Small}_{i} \cdot P A_{-} 5-6_{i}+\delta_{4} I_{i} \cdot$ Medium $_{i} \cdot P A_{-} 9-10_{i}+\delta_{5} I_{i} \cdot$ Medium $_{i} \cdot P A_{-} 7-$ $8_{i}+\delta_{6} I_{i} \cdot$ Medium $_{i} \cdot P A_{-} 5-6_{i}+\delta_{7} I_{i} \cdot$ Large $_{i} \cdot P A_{-} 9-10_{i}+\delta_{8} I_{i} \cdot$ Large $_{i} \cdot P A_{-} 7-8_{i}+\delta_{9} I_{i} \cdot$ Large $_{i} \cdot P A_{-} 5-6_{i}+\delta_{10} L S_{i} \cdot$ Small $_{i} \cdot P A_{-} 9-10_{i}+\delta_{11} L S_{i} \cdot \operatorname{Small}_{i} \cdot P A_{-} 7-8_{i}+\delta_{12} L S_{i} \cdot$ Small $_{i} \cdot$ $P A_{-} 5-6_{i}+\delta_{13} L S_{i} \cdot$ Medium $_{i} \cdot P A_{-} 9-10_{i}+\delta_{14} L S_{i} \cdot$ Medium $_{i} \cdot P A_{-} 7-8_{i}+\delta_{15} L S_{i} \cdot$ Medium $_{i} \cdot$ $P A_{-} 5-6_{i}+\delta_{16} L S_{i} \cdot \operatorname{Large}_{i} \cdot P A_{-} 9-10_{i}+\delta_{1} 7 L S_{i} \cdot$ Large $_{i} \cdot P A_{-} 7-8_{i}+\delta_{18} L S_{i} \cdot$ Large $_{i}$. $P A_{-} 5-6_{i}+\varepsilon_{i}$

Thus, in Equation 2, we use dummy variables for different strategic combinations. In addition to three order size categories (Small, Medium and Large), we also distinguish independent orders $(I)$ from split orders.

However, as a split order, by definition (see Section 4.3), is immediately followed by an identical limit order submission, we define:

- $\quad L S$ : the last split order in an order-splitting sequence.

Further, to ensure a sufficient number of observations for each dummy variable, we use three combinations of price aggressiveness ( $P A \_9-10, P A \_7-8$ and $\left.P A \_5-6\right)$. We use the same control variables as in Equation 1.

\subsection{Results of the Extended Model}

The results, which are consistent and strongly significant for both currency pairs, are shown in Table 4. 
Table 4: Results (Equation 2)

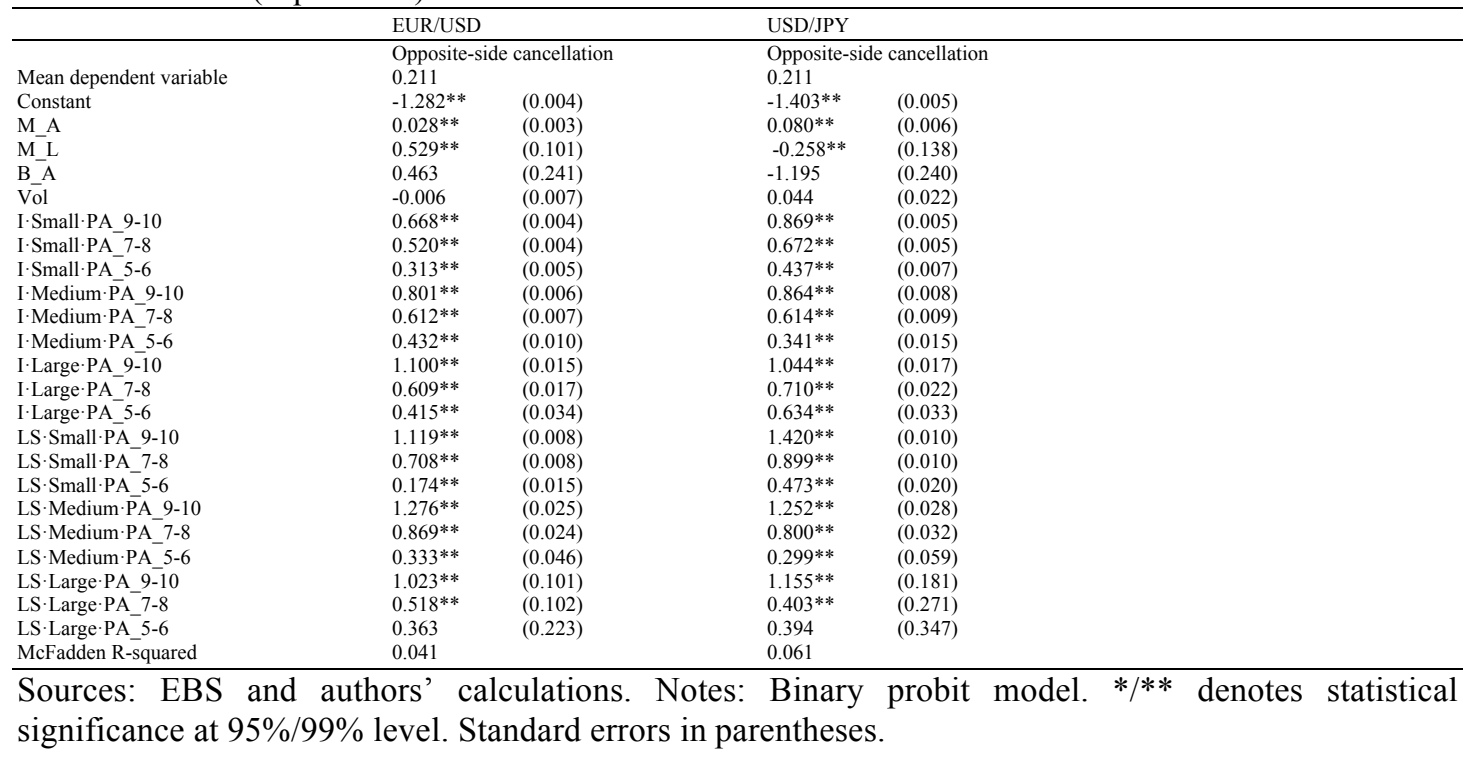

Three observations are notable.

First, except for large, relatively non-aggressive split-orders (which account for less than $0.003 \%$ of all orders), the results are consistent and strongly significant for all dummy variable combinations for both currency pairs. Information-rich orders induce opposite-side cancellations.

Second, for all order sizes, a higher level of price aggressiveness increases the probability of an immediate cancellation from the opposite side of the limit order book. However, the relationship does not consistently hold the other way round (i.e. given the level of price aggressiveness, a larger order does not necessarily increase the probability).

Third, small and medium-sized limit order submissions (of corresponding sizes and levels of price aggressiveness) completing an order-splitting strategy are more likely to trigger an immediate opposite-side limit order cancellation than independent limit order submissions. Such orders are more likely to trigger opposite-side cancellations than any other orders types.

Our results show that medium-sized and large orders, as a well as split orders, have a tendency to be more aggressive than small orders in the EUR/USD and USD/JPY FX spot markets on EBS. From the perspective of price aggressiveness, we could deduce 
that human traders (which are more inclined to submit medium-sized or large orders) are more likely to submit orders, which are intended to be executed. Such orders increase the probability of an immediate opposite-side cancellation if they are within three pips from the prevailing best bid-ask spread. For even higher levels of price aggressiveness, the probability increases significantly. However, small and relatively aggressive orders, which constitute the vast majority of the limit order submissions on the platform, show the same tendency. What is more, a sequence of algorithmic split orders increases the probability of opposite-side cancellations too (and more so than medium-sized or large orders) - despite being designed to prevent front-running by others.

\section{$5 \quad$ Pinging}

\subsection{Detecting Potential Ping Orders}

In Section 4, we observed that from the perspective of price aggressiveness, the EUR/SEK, USD/RUB and USD/TRY markets appear less susceptible to spoofing tactics than the major currency pairs. Furthermore, despite mainly containing sizeable orders, order-splitting strategies seem to be absent in the USD/RUB dataset. The EUR/SEK and USD/TRY datasets, by contrast, consists of virtually only minimumsized orders of 1 million. However, a fundamental difference lies in the choice of a trading platform in the decentralised FX market. EBS is the most widely used electronic trading platform for EUR/USD and USD/JPY. Reuters Matching, however, is the platform of choice for EUR/SEK and USD/TRY, and USD/RUB tends to be more liquid on MOEX. Our findings, therefore, should not be read as a suggestion that less liquid currencies are less susceptible to spoofing. Instead, the relative liquidity and competitiveness on trading venue might be critical. Ultimately, a spoof order is less likely to be successful on a secondary or tertiary trading platform where trading volume is lower; the best bid-ask spread wider, and other traders less likely to pay attention.

Nonetheless, during the active Swedish, Russian and Turkish FX trading hours, a limit order of at least 1 million was submitted approximately every two seconds on 
EBS. If the trading platform is less relevant, what could explain this continuous activity? This automatically leads to a question regarding the intent of these limit order submissions. Are they simply intended to ping the market for information?

Similarly to limit orders in general, ping orders are not intended to be executed immediately. Furthermore, as with spoof orders, ping orders are also (typically) intended to be cancelled, rather than executed. However, in contrast to spoof orders, they are not intended to trigger a reaction by other traders. If nothing happens, the order is immediately cancelled. If something happens, the trader directly obtains extracts some (potentially valuable) information about the state of the market. We cannot establish, for sure, whether a particular limit order should be classified as a ping order. Indeed, arguably the most significant hurdle for regulators and lawmakers striving to crack down on spoofing is the difficulty in obtaining evidence of intent with regards to the limit order submission process. Nonetheless, the journey from the time of submission to the time of cancellation of a limit order can provide us with useful insights into the prevalence of pinging in a specific market. After all, more than $99 \%$ of the limit order submissions in our original dataset were cancelled.

Here, it is useful to draw on the theoretical concept of non-execution risk and freeoption risk. By studying the limit order book immediately before the limit order submission, as well as immediately before its cancellation, we can establish which (if any) of the two risks is more important for traders. This, in turn, enables us to investigate the prevalence of pinging.

To do so, we first calculate the best bid and ask prices at the time of each limit order submission and cancellation. We also quantify the outstanding limit order volume at the best bid and ask prices, respectively, at the time of each entry to, and exit from, the market. Using the Trader IDs and the timestamps, we then calculate the change (if any) of the market price and volume during the lifetime of each order.

We classify an order as a 'non-execution order' $(N E O)$ if the best market price of a limit buy/sell order cancellation $[L B C / L S C]$ is higher/lower compared to immediately before the limit buy/sell order submission $[L B S / L S S]$. However, a trader having submitted a limit order might also anticipate that a change in market liquidity would 
have a subsequent impact on the future market price. Thus, if the best market price for a limit buy/sell order is unchanged immediately prior to the cancellation of the order compared to immediately prior to the submission of the order, but the relative volume $(R V)$ at the best market price has decreased/increased, we also classify the order as a non-execution order. We define relative volume as the total amount $(A)$ of outstanding limit buy orders at the best market bid price minus the total amount of outstanding limit sell orders at the best market ask price, immediately before to the $i$ th limit order submission. If the opposite is true, we classify the order as a 'free-option order' $(F O O)$. Finally, if the best market price, as well as the relative volume, for a limit order is unchanged immediately before the cancellation of the order compared to immediately before the submission of the order, we deduce that the order is a 'potential ping order' $(P P O)$. Hence:

- NEO:

$$
\begin{aligned}
& \text { ○ } \quad p_{L B C i-1}^{b m} \geq p_{L B S i-1}^{b m} \text { and } p_{L S C i-1}^{b m} \leq p_{L S S i-1}^{b m} \\
& \text { ○ } R V^{L B C i}<R V^{L B S i} \text {, } \\
& \text { where } R V^{L B C i}= \\
& \sum_{j=t(0)}^{j=t(L B C i-1)} A_{j}^{b b}+ \\
& \sum_{j=t(0)}^{j=t(L B C i-1)} A_{j}^{b a} \text { and } R V^{L B S i}=\sum_{j=t(0)}^{j=t(L B S i-1)} A_{j}^{b b}-\sum_{j=t(0)}^{j=t(L B S i-1)} A_{j}^{b a} \\
& \text { and } \\
& \text { ○ } R V^{L S C i c}>R V^{L S S i c} \text {, } \\
& \text { where } R V^{\text {LSCic }}= \\
& \sum_{j=t(0)}^{j=t(L S C i-1)} A_{j}^{b b}+ \\
& \sum_{j=t(0)}^{j=t(L S C i-1)} A_{j}^{b a} \text { and } R V^{L S S i c}=\sum_{j=t(0)}^{j=t(L S S i-1)} A_{j}^{b b}-\sum_{j=t(0)}^{j=t(L S S i-1)} A_{j}^{b a}
\end{aligned}
$$

- FOO:

$$
\begin{aligned}
\circ & p_{L B C i-1}^{b m} \leq p_{L L S S i-1}^{b m} \text { and } p_{L S C i-1}^{b m} \geq p_{L S S i-1}^{b m}, \\
\circ \quad & R V^{L B C i}>R V^{L B S i}, \\
& \text { where } R V^{L B C i}= \\
& \sum_{j=t(L B C i-1)}^{j=t(0)} A_{j}^{b b}+ \\
& \sum_{j=t(0 B C i-1)}^{j=t(0)} A_{j}^{b a} \text { and } R V^{L B S i}=\sum_{j=t(0)}^{j=t(L B S i-1)} A_{j}^{b b}-\sum_{j=t(0)}^{j=t(L B S i-1)} A_{j}^{b a} \\
& \text { and } \\
& R V^{L S C i c}<R V^{L S S i c}, \\
& \text { where } R V^{L S C i c}= \\
& \sum_{j=t((0) i-1)}^{j=t(0)} A_{j}^{b b}+ \\
& \sum_{j=t(L S C i-1)}^{j=t(0)} A_{j}^{b a} \text { and } R V^{L S S i c}=\sum_{j=t(0)}^{j=t(L S S i-1)} A_{j}^{b b}-\sum_{j=t(0)}^{j=t(L S S i-1)} A_{j}^{b a} .
\end{aligned}
$$

- $P P O$ :

If otherwise, where $\mathrm{LBS}_{\mathrm{i}-1}\left[\mathrm{LSS}_{\mathrm{i}-1}\right]$ is the time when the $\mathrm{i}-1$ th limit buy [sell] order enters the market and $\mathrm{LBC}_{\mathrm{i}-1}\left[\mathrm{LSC}_{\mathrm{i}-1}\right]$ is the time when the $\mathrm{i}-1$ th limit buy [sell] order exits from the market. 
Limit orders might, of course, be cancelled for a number of reasons. As stated previously, neither the intent behind the submission nor the cancellation can be determined with certainty without consultation with the trader in question. However, this methodology allows us to deduce whether limit orders, one average and depending on their characteristics, are cancelled because the market moves closer or further away. Importantly for the discussion below, it also enables us to distinguish the proportion of limit orders, which are cancelled before any change in price or volume has taken place.

Table 5 shows the results according to the order type (size and order-splitting) along with the average level of price aggressiveness.

Table 5: Limit order cancellations

\begin{tabular}{|c|c|c|c|c|c|c|}
\hline Currency pair & Order type & Limit orders & Agg & NEO & FOO & PPO \\
\hline \multirow{7}{*}{ EUR/USD } & Small (I) & $1,001,329$ & 6.58 & $47.56 \%$ & $42.01 \%$ & $10.43 \%$ \\
\hline & Small (S) & 226,759 & 8.28 & $54.75 \%$ & $39.10 \%$ & $6.16 \%$ \\
\hline & Medium (I) & 152,178 & 7.12 & $48.43 \%$ & $41.65 \%$ & $9.91 \%$ \\
\hline & Medium (S) & 19,166 & 8.36 & $53.32 \%$ & $40.87 \%$ & $5.81 \%$ \\
\hline & Large (I) & 18,925 & 8.19 & $69.44 \%$ & $26.41 \%$ & $4.15 \%$ \\
\hline & Large (S) & 1,316 & 9.13 & $69.22 \%$ & $27.66 \%$ & $3.12 \%$ \\
\hline & All & $1,419,673$ & 6.96 & $49.19 \%$ & $41.27 \%$ & $9.54 \%$ \\
\hline \multirow[t]{7}{*}{ USD/JPY } & Small (I) & 526,027 & 6.71 & $47.83 \%$ & $40.65 \%$ & $11.52 \%$ \\
\hline & Small (S) & 146,403 & 8.14 & $55.54 \%$ & $37.62 \%$ & $6.84 \%$ \\
\hline & Medium (I) & 87,000 & 6.72 & $48.40 \%$ & $39.69 \%$ & $11.91 \%$ \\
\hline & Medium (S) & 14,050 & 7.10 & $48.53 \%$ & $43.02 \%$ & $8.45 \%$ \\
\hline & Large (I) & 13,333 & 6.85 & $56.60 \%$ & $28.82 \%$ & $14.58 \%$ \\
\hline & Large (S) & 439 & 8.44 & $64.46 \%$ & $25.74 \%$ & $9.79 \%$ \\
\hline & All & 787,252 & 6.99 & $49.50 \%$ & $39.81 \%$ & $10.69 \%$ \\
\hline \multirow[t]{7}{*}{ EUR/SEK } & Small (I) & 52,840 & 6.68 & $34.24 \%$ & $14.87 \%$ & $50.89 \%$ \\
\hline & Small (S) & - & - & - & - & - \\
\hline & Medium (I) & - & - & - & - & - \\
\hline & Medium (S) & - & - & - & - & - \\
\hline & Large (I) & - & - & - & - & - \\
\hline & Large (S) & - & - & - & - & - \\
\hline & All & 52,840 & 6.68 & $34.24 \%$ & $14.87 \%$ & $50.89 \%$ \\
\hline \multirow[t]{7}{*}{ USD/RUB } & Small (I) & 619 & 6.40 & $9.69 \%$ & $3.23 \%$ & $86.91 \%$ \\
\hline & Small (S) & 4 & 6.00 & $0.00 \%$ & $25.00 \%$ & $75.00 \%$ \\
\hline & Medium (I) & 5,283 & 6.15 & $5.94 \%$ & $1.25 \%$ & $92.81 \%$ \\
\hline & Medium (S) & 62 & 7.45 & $58.06 \%$ & $1.61 \%$ & $40.32 \%$ \\
\hline & Large (I) & 25,569 & 7.33 & $18.23 \%$ & $4.62 \%$ & $77.16 \%$ \\
\hline & Large (S) & 500 & 7.11 & $18.20 \%$ & $2.40 \%$ & $79.40 \%$ \\
\hline & All & 32,037 & 7.11 & $16.11 \%$ & $4.00 \%$ & $79.89 \%$ \\
\hline \multirow[t]{7}{*}{ USD/TRY } & Small (I) & 38,610 & 7.17 & $54.05 \%$ & $25.09 \%$ & $20.86 \%$ \\
\hline & Small (S) & 66 & 9.20 & $59.09 \%$ & $33.33 \%$ & $7.58 \%$ \\
\hline & Medium (I) & 12 & 8.33 & $41.67 \%$ & $58.33 \%$ & $0.00 \%$ \\
\hline & Medium (S) & - & - & - & - & - \\
\hline & Large (I) & - & - & - & - & - \\
\hline & Large (S) & - & - & - & - & - \\
\hline & All & 38,688 & 7.18 & $54.06 \%$ & $25.11 \%$ & $20.83 \%$ \\
\hline
\end{tabular}

Sources: EBS and authors' calculations. Notes: $\mathrm{S}=$ Split, $\mathrm{I}=$ Independent, Agg $=$ Price aggressiveness on a scale from 1 to $10, \mathrm{NEO}=$ Non-execution order, $\mathrm{FOO}=$ Free-option order, $\mathrm{PPO}=$ Potential ping order.

The concept of non-execution risk and free-option risk is well established in the theoretical market microstructure literature. Market participants also report that traders behave differently depending on their "urgency" to transact. However, we are not aware of any attempts to quantify such perceived risks (let alone the prevalence of 
pinging) in the FX markets - presumably due to the lack of complete limit order book data. With this in mind, the following three observations should be highlighted.

First, when studying the likely causes of limit order cancellations for the five currency pairs, non-execution risk appears to be more important than free-option risk. In the EUR/USD market, for instance, approximately $49 \%$ of limit orders are cancelled following (or: as a result of) an adverse move in the market, and only $41 \%$ after a favourable change. The differences for the other four currency pairs are somewhat larger, with up to $54 \%$ versus $25 \%$ for USD/RUB.

Second, in the EUR/USD and USD/JPY markets, orders that do not fall into the categories of being small or independent are most likely to be non-execution orders. Furthermore, non-execution orders are consistently more aggressive than free-option orders. There is a plausible explanation for this. At the time of submission, we might expect traders concerned about non-execution (rather than free-option) risk to be inclined to submit relatively more competitive orders. The results support this intuition. The findings could also be interpreted in conjunction with the results in Section 4.5. Put together, this would suggest that relatively large limit orders submitted by human traders, and split orders submitted by algorithmic traders, are more likely to be orders which are intended to be executed.

Third, around $10 \%$ of all EUR/USD and USD/JPY orders are cancelled before neither the best market price nor the volume at the best market price has changed. In the USD/TRY market, potential ping orders account for around 20\%. In the EUR/SEK and USD/RUB, the share is remarkably high: $50 \%$ and $80 \%$, respectively. Logically, too, limit order submissions in these three markets are also more likely to immediately be followed by a same-side cancellation (see Table 3). For EUR/SEK and USD/TRY, the probability is over $50 \%$, compared to $35 \%$ for USD/RUB and $31-$ $32 \%$ for the major currency pairs. In sum, it appears as if limit orders for the currency pairs where EBS is used as a secondary platform are much more likely to be cancelled immediately or, at least, before the market has moved. 


\subsection{The Lifetime of Limit Orders}

So far, we have deduced that the three currency pairs where EBS is used a secondary platform (EUR/SEK, USD/RUB and USD/TRY) appear to be less sensitive to spoofing tactics - perhaps because the liquidity on EBS is relatively thin. At the same time, however, they contain many (and a high proportion) of limit orders, which are cancelled before the best price has moved - or even immediately after submission.

One could, plausibly, argue that less active markets automatically result in a relatively higher proportion of orders that would be classified as potential ping orders. Logically, the time window "when nothing happens" is longer and, therefore, more likely to induce traders to cancel and reassess the order submission strategy. If so, the 'lifetime' of limit orders in the less liquid currency pairs should, on balance, be longer. By using the ID and timestamp of each limit order submission, and matching it with the corresponding ID and timestamp of the cancellation of it, we can calculate the lifetime of the limit orders in our dataset.

Table 6: Lifetime of limit order submissions

\begin{tabular}{|c|c|c|c|c|c|c|c|c|c|c|}
\hline & EUR/USD & & USD/JPY & & EUR/SEK & & USD/RUB & & USD/TRY & \\
\hline Order type & Frequency & Lifetime & Frequency & Lifetime & Frequency & Lifetime & Frequency & Lifetime & Frequency & Lifetime \\
\hline Small (I) & $70.53 \%$ & $00: 39.7$ & $66.82 \%$ & 01:05.1 & $100.00 \%$ & $00: 16.2$ & $1.93 \%$ & $13: 21.6$ & $99.80 \%$ & $00: 23.5$ \\
\hline Small (S) & $15.97 \%$ & $00: 28.8$ & $18.60 \%$ & $00: 50.4$ & $0.00 \%$ & - & $0.01 \%$ & $00: 00.3$ & $0.17 \%$ & $00: 14.8$ \\
\hline Medium (I) & $10.72 \%$ & 01:05.2 & $11.05 \%$ & $01: 51.4$ & $0.00 \%$ & - & $16.49 \%$ & 00:01.3 & $0.03 \%$ & $03: 42.6$ \\
\hline Medium (S) & $1.35 \%$ & $00: 29.0$ & $1.78 \%$ & $00: 41.0$ & $0.00 \%$ & - & $0.19 \%$ & $00: 00.1$ & $0.00 \%$ & - \\
\hline Large (I) & $1.33 \%$ & $06: 07.8$ & $1.69 \%$ & $13: 22.7$ & $0.00 \%$ & - & $79.81 \%$ & $00: 02.1$ & $0.00 \%$ & - \\
\hline Large (S) & $0.09 \%$ & $00: 31.0$ & $0.06 \%$ & $00: 51.7$ & $0.00 \%$ & - & $1.56 \%$ & 00:00.1 & $0.00 \%$ & - \\
\hline All & $100.00 \%$ & $00: 44.9$ & $100.00 \%$ & $01: 19.5$ & $100.00 \%$ & $00: 16.2$ & $100.00 \%$ & $00: 17.4$ & $100.00 \%$ & $00: 23.6$ \\
\hline PPO & $9.54 \%$ & $00: 03.4$ & $10.69 \%$ & 00:05.3 & $50.89 \%$ & 00:08.0 & $79.89 \%$ & $00: 00.5$ & $20.83 \%$ & $00: 15.3$ \\
\hline PPO 0.5 & $5.25 \%$ & $<00: 00.5$ & $5.40 \%$ & $<00: 00.5$ & $32.10 \%$ & $<00: 00.5$ & $78.03 \%$ & $<00: 00.5$ & $4.72 \%$ & $<00: 00.5$ \\
\hline
\end{tabular}

Sources: EBS and authors' calculations. Notes: $\mathrm{S}=$ Split, I = Independent, NEO = Non-execution order, FOO $=$ Free-option order, PPO $=$ Potential ping order, PPO_0.5 $=$ Potential ping order with a lifetime shorter than 0.5 seconds.

The FX spot market is famously fast-paced. Indeed, the average lifetime of limit orders in our dataset ranges from just 16.2 seconds in the EUR/SEK market to 1 minute and 19.5 seconds in the USD/JPY market (see Table 6). However, this includes orders that stay in the limit order book for a very long time - sometimes up to several hours. The median lifetime is considerably shorter. Two important patterns are notable.

First, in the EUR/USD and USD/JPY markets, medium-sized and, in particular, large limit orders have a considerably longer lifetime than small orders. Likewise, for 
corresponding order sizes, independent orders remain in the limit order book for a notably shorter time than split orders. Previous studies show that markets populated with algorithmic and high-frequency traders display a very high number of limit order submissions and cancellations - and their lifetime tends to be very short (Harris and Hasbrouck, 1996; Hasbrouck and Saar, 2002; Hollifield et al., 2004). The results can, therefore, be viewed from the perspective of human versus non-human trading. The average lifetime of human limit order submissions is likely to be longer - by being slower and less able to, repeatedly, cancel and resubmit orders. The short lifetime of split-orders (submitted by non-human traders) and long lifetime of medium-sized and large orders (where human traders presumably dominate) confirm this.

Second, the average lifetime of limit orders for the three currency pairs where EBS is used as a secondary platform (EUR/SEK, USD/RUB and USD/TRY) is considerably shorter. This is a consequence of the high proportion of potential ping orders, whose average lifetime ranges from just 0.5 seconds for USD/RUB to 15.3 seconds for USD/TRY. Given that these orders are cancelled before the state of the market has changed, the lifetime of these orders will, of course, be shorter on balance.

In debates about fairness (or the lack of it) surrounding high-frequency trading, the human inability to fast enough is often brought up as an argument among critics. Indeed, psychologists estimate that it takes approximately 300-400 milliseconds for a human being to blink (Geiger and Mamudi, 2014). Using 0.5 seconds as a benchmark, we can calculate the proportion of limit order submissions that are not only cancelled before the state of the market has changed, but also before a human trader has had the opportunity to blink. As can be seen from Table 6, almost one-third of EUR/SEK FX spot limit orders on EBS are classified as potential ping orders with a lifetime of shorter than 0.5 seconds (PPO_0.5). A remarkable 97.7\% of potential ping orders in the USD/RUB market stay in the limit order book for less than half a second.

\section{Concluding Discussion}

In this paper, we have explored the susceptibility of FX spot markets to limit order submission strategies either intended to create a false impression of the state of the 
market ('spoof orders') or to extract hidden information in the market ('ping orders'). To do so, we have studied 2,330,480 limit order submissions and cancellations with a total value of more than $\$ 3$ trillion, having obtained a full limit order book dataset provided by EBS.

The aim of a spoof order is to cause other traders to react as if genuine price-moving information has entered the market. Regarding susceptibility to spoof orders, we find that the EUR/USD and USD/JPY FX spot markets are highly sensitive to information-rich limit order submissions. A larger order, or a higher level of price aggressiveness, increases the probability of an immediate cancellation from the opposite side of the limit order book. However, given the level of price aggressiveness, a larger order does not necessarily increase the probability of such a reaction. Thus, price aggressiveness, it seems, is more critical than size. Interestingly, a sequence of algorithmic split orders designed to prevent front-running by hiding the 'true' size increases the probability of opposite-side cancellations (and more so than medium-sized or large orders).

The link between aggressive limit order submissions and immediate opposite-side cancellations is considerably less robust for EUR/SEK, USD/RUB and USD/TRY. What is more, we detect hardly any strategies designed to prevent front-running, where EBS is used as a secondary electronic trading platform. For spoofing tactics to be successful, market participants ought to react with some kind of predictability: namely to immediately exit, rather than immediately enter, the opposite side of the limit order book. Thus, our empirical results suggest that spoofing tactics do not necessarily need to involve large and non-aggressive limit orders (as indicated in the equity markets literature) or be more likely to succeed in illiquid markets (as suggested by market participants). ${ }^{14}$ Instead, deceptive trading strategies intended to create a false impression of the market might evolve very differently depending on the chosen electronic trading venue and the market conventions associated with it.

Furthermore, our results indicate that potential ping orders seem to be more prevalent in FX spot markets, which are thinner on EBS. Depending on the criteria used, such

\footnotetext{
${ }^{14}$ Lee et al. (2012), using stock market data from the Korean Exchange (KRX) from 2001-02, find that a spoof order would typically be not only large, but also relatively non-aggressive.
} 
orders could account for up to one-third in the EUR/SEK market and an astounding $80 \%$ in the USD/RUB market. Regardless if such limit orders are used to front-run the market (which obviously would be serious), the question is whether they add liquidity, act to create an artificial view of the state of the market - or irrelevant? Suppose no potential ping orders were submitted to the limit order book. Would the liquidity be notably different?

To test this, let us exclude all potential ping orders from the dataset, and recalculate both liquidity proxies defined in Section 4.1: the average bid-ask spread $\left(B_{-} A\right)$ and the average volume at the best bid-ask spread $\left(M_{-} L\right)$. Table 7 shows a comparison.

Table 7: Market Liquidity with and without potential pinging

\begin{tabular}{llllll}
\hline & EUR/USD & USD/JPY & EUR/SEK & USD/RUB & USD/TRY \\
\hline All orders & & & & & \\
M_L (mio) & 16.17 & 17.14 & 1.90 & 15.27 & 2.17 \\
B_A (\%) & 0.0083 & 0.0134 & 0.1327 & 0.0514 & 0.0559 \\
\hline All orders (excl. PPO) & 16.90 & 18.06 & 2.00 & 11.22 & 2.14 \\
M_L (mio) & 0.0127 & 0.0157 & 0.1590 & 0.1676 & 0.0600 \\
\hline B_A (\%) & &
\end{tabular}

Sources: EBS and authors' calculations.

At the outset, we can see that the bid-ask spread is exceptionally tight for EUR/USD and USD/JPY (0.0083\% and $0.0127 \%$, respectively). This is consistent with EBS being the leading trading platform for the two most actively traded currency pairs in the world. What is more, an average of 16-17 million worth of limit orders are tradable at this spread. The others are less liquid (by not only being smaller currency pairs but also less actively traded on EBS). Hence, their bid-ask spread is considerably wider, and the volume-based liquidity proxy is lower - except for USD/RUB, which is boosted by the large average order size.

Excluding the potential ping orders (which account for approximately 10\%, 10\%, $20 \%$ and $50 \%$ for EUR/USD, USD/JPY, USD/TRY and EUR/SEK, respectively) does not change the result dramatically. The spread is only marginally wider in absolute and percentage terms. The liquidity in the USD/RUB market, however, is clearly affected when excluding potential ping orders. The bid-ask spread is more than three times wider, and the average volume lower. Thus, one could argue that such orders contribute to the liquidity. On the other hand, given the vast proportion of 
such orders in, say, the EUR/SEK market, one could also plausibly claim that their marginal contribution is minimal.

However, the two liquidity measures above only capture marketable and at-the-quote orders. Traders respond to changes in the limit order book as a whole, which includes (potential ping) orders submitted outside the prevailing best bid-ask spread. We, therefore, need to take into account the flickering activity that takes place within the limit order book as a whole. Suppose each 24-hour trading day is divided into 15minute time buckets and that each new limit order submission is allocated to a particular bucket according to its timestamp. Each limit order is then weighted according to its size and lifetime (where the maximum lifetime is capped at 15 minutes to avoid overstating the contribution of orders that stay in the book for several hours). Thus, a 2-million limit order remaining in the book for 15 minutes contributes to the liquidity by 2 million, a 1-million order staying for 7.5 minutes by 0.5 million, and so on.

Figure 3 shows the results for EUR/SEK. Using this liquidity contribution proxy, approximately $€ 3-4$ million is added to the limit order book during every 15 -minute time bucket, apart from when the Swedish domestic market is closed. Although potential ping orders $(P P O)$ do not dominate overall, they occasionally stand for a significant proportion of the liquidity added. The impact of potential ping orders with a lifetime of shorter than 0.5 seconds $\left(P P O \_0.5\right)$ is barely visible - despite accounting for $32 \%$ of all limit order submissions. 
Figure 3: Liquidity contribution, EUR/SEK, 8.9.2010 (21:00:00 GMT) - 13.9.2010 (21:00:00 GMT)

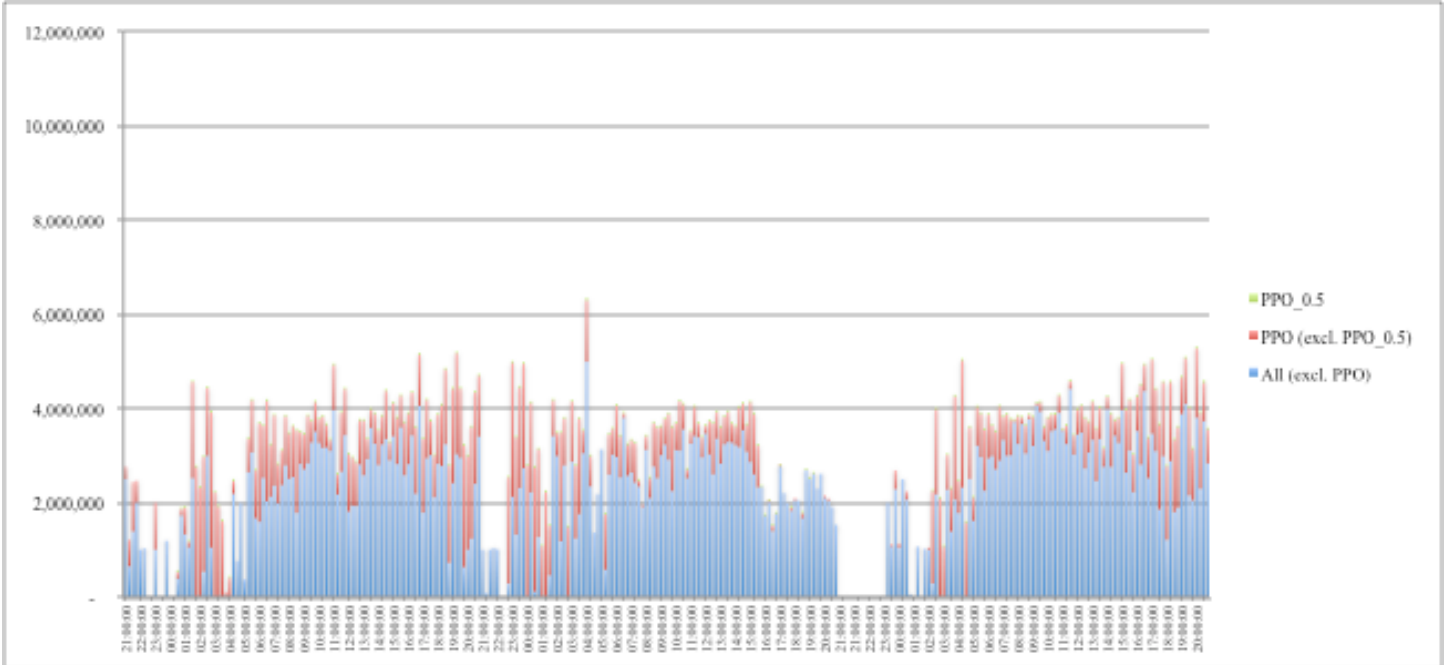

Sources: EBS and authors' calculations. Note: the figure excludes the weekend: 10.9.2010 (21:00:00 GMT) - 12.9.2010 (21:00:00 GMT).

The results for USD/RUB, however, are totally different - despite containing approximately the same number, and, importantly, considerably larger, limit orders (Figure 4). Seen from this perspective, the liquidity of the USD/RUB market appears to be remarkably thin and often made up by potential ping orders that disappear within a flash.

Figure 4: Liquidity contribution, USD/RUB, 8.9.2010 (21:00:00 GMT) - 13.9.2010 (21:00:00 GMT)

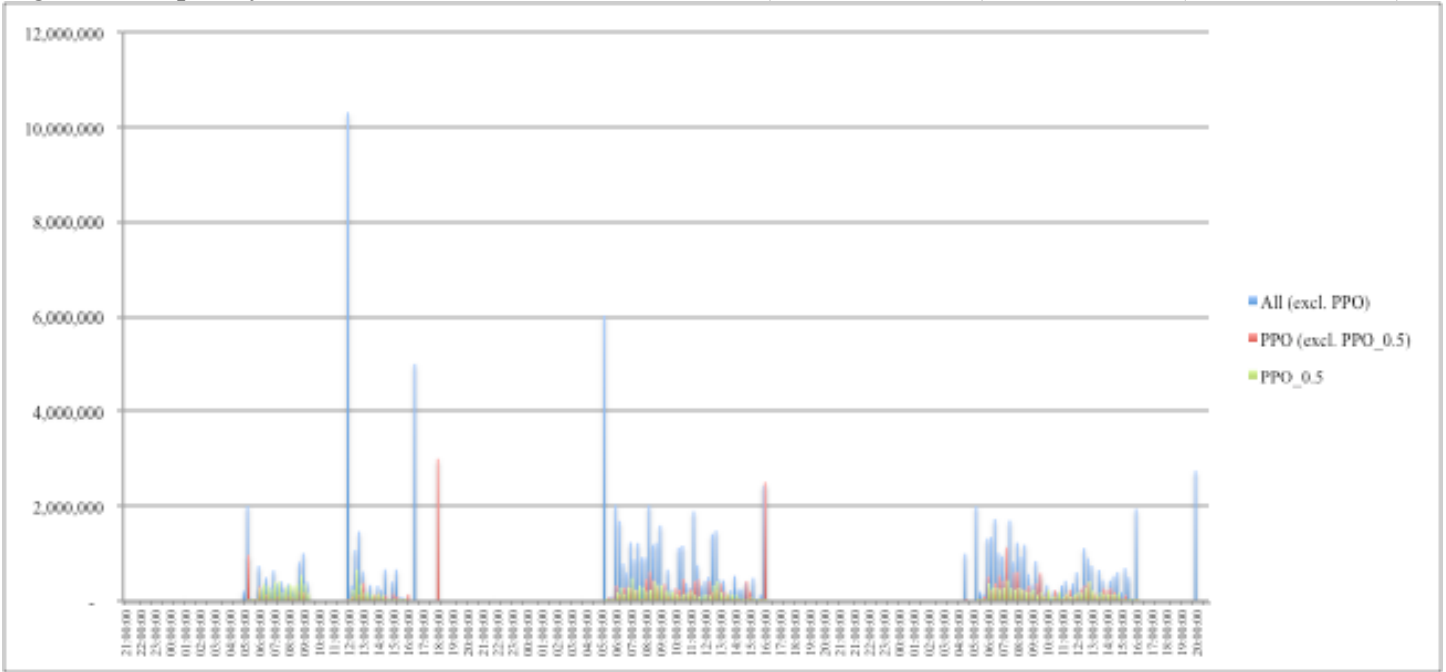

Sources: EBS and authors' calculations. Note: the figure excludes the weekend: 10.9.2010 (21:00:00 GMT) - 12.9.2010 (21:00:00 GMT).

In August 2017, Janet Yellen, former Chair of the U.S. Federal Reserve, stated that "algorithmic traders ... are a larger presence in various markets than previously, and the willingness of these institutions to support liquidity in stressful conditions is uncertain" (Federal Reserve, 2017). Notably, so-called "flash crashes" are, almost by 
definition, linked to electronic markets. Compared to equity markets, algorithmic trading is relatively new in FX markets. However, this has changed dramatically during the last decade, and the trend is unlikely to reverse following the Forex scandal. The reform processes induced by regulatory agencies and the industry itself have focussed on imposing stricter communication rules between traders, salespeople and the banks' clients, as well as between competing traders at market-making banks. Importantly, a shift towards electronic trading is increasingly encouraged also as a means to minimise the likelihood of human misconduct and conflict of interest. In February 2018, the Bank of England and the UK Financial Conduct Authority issued a joint statement proposing to bring algorithmic trading in the wholesale markets (mentioning FX in particular) under their supervision (Bank of England 2018; FCA, 2018). Importantly, too, the new voluntary Global FX Code stresses that 'market participants should not [...] create orders with the intention of disrupting market functioning or hindering the price discovery process, including undertaking actions designed to result in a false impression of market price, depth, or liquidity' (BIS, 2017). Put together; these developments are likely to necessitate a more in-depth understanding of limit order books involving FX and algorithms - and the growing importance of non-human traders in OTC markets. This undoubtedly includes the susceptibility to, and prevalence of, trading tactics such as spoofing or pinging. 


\section{References}

Allen, F., Gale, F., 1992. Stock-Price Manipulation. The Review of Financial Studies, $5(3), 502-529$.

Bank of England, 2018. Algorithmic trading, Consultation Paper CP5/18, February. Available from: https://www.bankofengland.co.uk/-/media/boe/files/prudentialregulation/consultationpaper/2018/cp518.pdf?la=en\&hash=89AB31B883DF430E36387BACCC93F15FC7A 75A4A [accessed 15 February 2018].

Barclay, M., Warner, J., 1993. Stealth Trading and Volatility: Which Trades Move Prices? Journal of Financial Economics, 34, 281-305.

Bershova, N., Rakhlin, D., 2013. High-frequency trading and long-term investors: A view from the buy-side. Journal of Investment Strategies, 2, 25-69.

Bertsimas, D., Lo, A., 1998. Optimal Control of Execution Costs. Journal of Financial Markets, 1 (1), 1-50.

Bessembinder, H., Panayides, M., Venkataraman, K., 2009. Hidden liquidity: An analysis of order exposure strategies in electronic stock market. Journal of Financial Economics, 94 (3), 361-383.

Biais, B., Hillion, P., Spatt, C., 1995. An Empirical Analysis of the Limit Order Book and the Order Flow on the Paris Bourse. Journal of Finance, 50 (5), 1655-1689.

BIS, 2011. High-frequency trading in the foreign exchange market.

BIS, 2013. Triennial Central Bank Survey - Foreign exchange turnover in April 2013: preliminary global results, September 2013. Available from:

http://www.bis.org/publ/rpfx13fx.pdf [accessed 17 May 2017].

BIS, 2016. Triennial Central Bank Survey - Foreign exchange turnover in April 2016, September 2016. Available from: http://www.bis.org/publ/rpfx16fx.pdf [accessed 17 May 2017].

BIS, 2017. FX Global Code: A set of global principles of good practice in the foreign exchange market. Available from: https://www.globalfxc.org/docs/fx_global.pdf [accessed 15 February 2018].

Bloomfield, R., O'Hara, M., Saar, G., 2015. Hidden Liquidity: Some New Light on Dark Trading. Journal of Finance, 70 (5), 2227-2274.

Brogaard, J., Hendershott, T., Riordan, R., 2014. High frequency trading and price discovery. Review of Financial Studies, 27 (8), 2267-2306.

CFTC, 2014. In the Matter of: JPMorgan Chase Bank, N.A. Respondent, CFTC Docket No. 15-04, 11 November. Available from: 
http://www.cftc.gov/idc/groups/public/@1renforcementactions/documents/legalpleadi ng/enfjpmorganorder111114.pdf [accessed 15 February 2018].

CFTC, 2018. CFTC Files Eight Anti-Spoofing Enforcement Actions against Three Banks (Deutsche Bank, HSBC \& UBS) \& Six Individuals, Press release, 29 January. Available from: http://www.cftc.gov/PressRoom/PressReleases/pr7681-18 [accessed 15 February 2018].

Chan, L., Lakonishok, J., 1995. The behavior of stock prices around institutional trades. Journal of Finance, 50 (4), 1147-1174.

Chou, R. K, Wang, Y-Y, 2009. Strategic order splitting, order choice, and aggressiveness: Evidence from the Taiwan futures exchange. Journal of Futures Markets, 29, 1102-1129.

Cheung, Y.-W., Chinn, M. D., 2001. Currency traders and exchange rate dynamics: a survey of the US market. Journal of International Money and Finance, 20 (4), 439471.

Cheung, Y.-W., Wong, C. Y.-P., 2000. A survey of market practitioners' views on exchange rate dynamics. Journal of International Economics, 51, 401-419.

Conrad, J., Wahal, S., Xiang, J., 2015. High-frequency quoting, trading, and the efficiency of prices. Journal of Financial Economics, 116, 271-291.

Cumming, D., Dannhauser, R., Johan, S., 2015. Financial market misconduct and agency conflicts: A synthesis and future directions. Journal of Corporate Finance, 34, $150-168$.

Cumming, D., Johan, S., Li, D., 2011. Exchange trading rules and stock market liquidity. Journal of Financial Economics, 99, 651-671.

Daníelsson , J., Luo, J., Payne, R., 2012. Exchange rate determination and intermarket order flow effects. European Journal of Finance, 18 (9), 823-840.

DFS, 2015. NYDFS announces Barclays to pay additional \$150 million penalty, terminate employee for automated, electronic trading misconduct, press release, 18 November. Available from: http://www.dfs.ny.gov/about/press/pr1511181.htm [accessed 15 February 2018].

DFS, 2017a. DFS fines BNP Paribas \$350 million for illegal, unsafe and unsound conduct in connection with BNPP's foreign exchange trading business, press release, 24 May. Available from: http://www.dfs.ny.gov/about/press/pr1705241.htm [accessed 15 February 2018].

DFS, 2017b. DFS fines Credit Suisse AG \$135 million for illegal, unsafe and unsound conduct in its foreign exchange trading business, press release, 13 November. Available from: http://www.dfs.ny.gov/about/press/pr1711131.htm [accessed 15 February 2018]. 
DOJ, 2016. Global Head of HSBC's Foreign Exchange Cash-Trading Desks Arrested for Orchestrating Multimillion-Dollar Front Running Scheme, press release, 20 July. Available from: https://www.justice.gov/opa/pr/global-head-hsbc-s-foreign-exchangecash-trading-desks-arrested-orchestrating-multimillion [accessed 15 February 2018].

Engle, R. F., Ferstenberg, R., Russell, J. R., 2012. Measuring and modeling execution cost and risk. Journal of Portfolio Management, 38 (2), 14-28.

Evans, M., Lyons, R., 2002. Order flow and exchange rate dynamics. Journal of Political Economy, 110, 170-180.

Evans, M., Lyons, R., 2005. Meese-Rogoff redux: Micro-based exchange-rate forecasting. American Economic Review Papers and Proceedings 95 (2), 405-414.

Fan, M., Lyons, R., 2003. Customer trades and extreme events in foreign exchange. In: Mizen, P. (Ed.) Monetary History, Exchange Rates and Financial Markets: Essays in Honor of Charles Goodhart. Northampton, MA: Edward Elgar, 160-179.

FCA, 2014. FCA fines five banks $£ 1.1$ billion for FX failings and announces industrywide remediation programme, Press release, 12 November. Available from: https://www.fca.org.uk/news/press-releases/fca-fines-five-banks-\%C2\%A311-billionfx-failings-and-announces-industry-wide [accessed 15 February 2018].

FCA, 2018. Algorithmic Trading Compliance in Wholesale Markets, February. Available from: https://www.fca.org.uk/publication/multi-firm-reviews/algorithmictrading-compliance-wholesale-markets.pdf [accessed 15 February 2018].

Federal Reserve, 2017. Financial Stability a Decade after the Onset of the Crisis, 25 August Available from:

https://www.federalreserve.gov/newsevents/speech/yellen20170825a.htm [accessed 15 February 2018].

FINMA, 2014. Foreign exchange trading at UBS AG: investigation conducted by FINMA, Report, 12 November. Available from:

https://www.finma.ch/en/news/2014/11/mm-ubs-devisenhandel-20141112/ [accessed 15 February 2018].

FINMA, 2015. Foreign exchange manipulation: FINMA issues six industry bans, Press release, 17 December. Available from: https://www.finma.ch/en/news/2015/12/20151217-mm-devisenhandel/ [accessed 15 February 2018].

Fong, K. Y. L., Liu, W. M., 2010. Limit order revisions. Journal of Banking and Finance, 34, 1873-1885.

Foucault, T., 1999. Order flow composition and trading costs in a dynamic limit order market. Journal of Financial Markets, 2, 99-134.

Foucault, T., Kadan, O., Kandel, E., 2005. Limit order book as a market for liquidity, Review of Financial Studies. 18 (4), 1171-1217. 
Geiger, K., Mamudi, S., 2014. High-Speed Trading Faces New York Probe Into Fairness, Bloomberg, 18 March. Available from:

https://www.bloomberg.com/news/articles/2014-03-18/high-speed-trading-said-toface-n-y-probe-into-fairness [Accesses 31 August 2018.

Froot, K. A., Ramadorai, T., 2005. Currency Returns, Intrinsic Value and Institutional-Investor Flow. Journal of Finance, 60, pp. 1535-1566.

Harris, L., 2013. What to do about high-frequency trading. Financial Analysts Journal, 69 (2), 6-9.

Harris, L., Hasbrouck, J., 1996. Market vs. Limit Orders: The SuperDOT Evidence on Order Submission Strategy. The Journal of Financial and Quantitative Analysis, 31 (2), 213-231.

Hasbrouck, J., Saar, G., 2002. Limit orders and volatility on a hybrid market. Working paper, New York University.

Hendershott, T., Jones, C. M., Menkveld, A. J., 2011. Does Algorithmic Trading Improve Liquidity? Journal of Finance, 66 (1), 1-33.

Hodges, J., 2018. Ex-HSBC FX Trader Wins Rare U.K. Order Blocking Extradition, Bloomberg, 31 July. Available from:

https://www.bloomberg.com/news/articles/2018-07-31/ex-hsbc-fx-trader-wins-u-korder-blocking-extradition-to-u-s [accessed 29 August 2018].

Hollifield, B., Miller, R. A., Sandås, P., 2004. Empirical Analysis of Limit Order Markets. Review of Economic Studies, 71 (4), 1027-1063.

Jarrow, R. A., 1994. Derivatives securities markets, market manipulation, bubbles, and option pricing theory. Journal of Financial and Quantitative Analysis, 29, 241246.

Keim, D., Madhavan, A., 1995. Anatomy of the trading process: empirical evidence on the behavior of institutional traders. Journal of Financial Economics, 37, 371-398.

Keim, D., Madhavan, A., 1996. The upstairs market for large-block transactions: analysis and measurement of price effects. Review of Financial Studies, 9 (1), 1-36.

King, M. R., Rime, D., 2010. The \$4 trillion question: what explains FX growth since the 2007 survey? BIS Quarterly Review, December 2010.

Lee, E. J., Eom, K. S., Park, K. S., 2012. Microstructure-based manipulation: Strategic behaviour and performance of spoofing traders. Journal of Financial Markets, 16, 227-252.

Liu, W-M., 2009. Monitoring and limit order submission risks. Journal of Financial Markets, 12, 107-141. 
Lo, I., Sapp, S. G., 2010. Order Aggressiveness and Quantity: How Are They Determined in a Limit Order Market? Journal of International Financial Markets, Institutions and Money, 20, 213-237.

Lyons, R. K., 1997. A simultaneous trade model of the foreign exchange hot potato. Journal of International Economics, 42, 275-298.

McGowan, M. J., 2010. The Rise of Computerized High Frequency Trading: Use and Controversy. Duke Law and Technology Review.

Menkhoff, L, 2010. High-frequency analysis of foreign exchange interventions: what do we learn? Journal of Economic Surveys, 24 (1), 85-112.

Moore, M., Schrimpf, A., Sushko, V., 2016. Downsized FX markets: causes and implications. BIS Quarterly Review, December.

Nikkei Asian Review, 2018. MUFG brokerage venture slammed for 'spoofing' bond market, 30 June. Available from: https://asia.nikkei.com/Business/Markets/MUFGbrokerage-venture-slammed-for-spoofing-bond-market [Accessed 29 August 2018].

Osler, C., 2003. Currency orders and exchange-rate dynamics: explaining the success of technical analysis. Journal of Finance, 58 (5), 1791-1819.

Osler, C., 2005. Stop-loss orders and price cascades in currency markets. Journal of International Money and Finance, 24 (2), 219-241.

Osler, C, Vandrovych, V., 2009. Hedge Funds and the Origins of Private Information in Currency Markets. Working Paper. Brandeis University.

Quereé, N., 2016. 'Spoofing' is not a crime, but should it be? The Times, 8 December. Available from: https://www.thetimes.co.uk/article/spoofing-is-not-acrime-but-should-it-be-5hq8pw875 [accessed 15 February 2018].

Payne, R., 2003. Informed trade in spot foreign exchange markets: an empirical investigation. Journal of International Economics, 61 (2), 307-329.

Peiers, B., 1997. Informed traders, intervention, and price leadership: a deeper view of the microstructure of the foreign exchange market. Journal of Finance, 52 (4), $1589-1614$

Pérold, A., 1988. The implementation shortfall: paper versus reality. Journal of Portfolio Management, 14, 4-9.

Pirrong, C., 2017. The economics of commodity market manipulation: A survey. Journal of Commodity Markets, 5, 1-17.

Rosu, I., 2009. A dynamic model of the limit order book. Review of Financial Studies, 22 (11), 4601-4641. 
Scopino, G., 2015. The (Questionable) Legality of High-Speed Pinging and Front Running in the Futures Market. Connecticut Law Review, 47.

Stenfors, A., 2018. Bid-Ask Spread Determination in the FX Swap Market:

Competition, Collusion or a Convention? Journal of International Financial Markets, Institutions and Money, 58, 78-97.

Stenfors, A., Lindo, D., 2018. Libor 1986-2021: the making and unmaking of 'the world's most important price'. Distinktion: Journal of Social Theory, 19 (2), 170-192.

Stenfors, A., Susai, M., 2018a. High-frequency Trading, Liquidity Withdrawal, and the Breakdown of Conventions in Foreign Exchange Markets. Journal of Economic Issues, 52 (2), 385-395.

Stenfors, A., Susai, M., 2018b. Liquidity Withdrawal in the FX Spot Market: A Cross-Country Study Using High-Frequency Data. Journal of International Financial Markets, Institutions and Money (forthcoming).

Vaughan, L., Finch, G., Choudhury, A., 2013. Traders Said to Rig Currency Rates to Profit Off Clients, Bloomberg, 12 June. Available from:

http://www.bloomberg.com/news/articles/2013-06-11/traders-said-to-rig-currencyrates-to-profit-off-clients [accessed 15 February 2018].

Yeo, W.Y., 2005. Cancellations of limit order. Working paper, National University of Singapore. 